\title{
The mixing transition in turbulent flows
}

\author{
By PAUL E. DIMOTAKIS \\ Graduate Aeronautical Laboratories, California Institute of Technology, Pasadena, CA 91125, USA
}

(Received 15 January 1999 and in revised form 15 May 1999)

Data on turbulent mixing and other turbulent-flow phenomena suggest that a (mixing) transition, originally documented to occur in shear layers, also occurs in jets, as well as in other flows and may be regarded as a universal phenomenon of turbulence. The resulting fully-developed turbulent flow requires an outer-scale Reynolds number of $R e=U \delta / v \gtrsim 1-2 \times 10^{4}$, or a Taylor Reynolds number of $R e_{T}=u^{\prime} \lambda_{T} / v \gtrsim 100-140$, to be sustained. A proposal based on the relative magnitude of dimensional spatial scales is offered to explain this behaviour.

\section{Introduction}

A correct description of turbulent mixing is particularly taxing on our understanding of turbulence; such a description relies on an account of the dynamics spanning the full spectrum of scales. Specifically, to describe the entrainment stage that is responsible for the engulfment of large pockets of irrotational fluid species into the turbulent flow region (Brown \& Roshko 1974), the large-scale flow structures need to be correctly described. Secondly, to describe the subsequent kinematic stirring process responsible for the large interfacial surface generation between the mixing species, the intermediate range of scales must be correctly accounted for. These are below the largest in the flow in size, but above the smallest affected by viscosity and molecular diffusivity. Finally, the dynamics at the smallest scales must be captured to describe the molecular mixing process itself. These three phases of turbulent mixing were identified as 'more or less distinct stages' in the description of mixing by Eckart (1948), who dubbed them as the initial, intermediate, and final stages, respectively. In the case of mixing of high Schmidt number $(S c \equiv v / \mathscr{D})$ fluids, i.e. fluids characterized by a molecular diffusivity, $\mathscr{D}$, much smaller than the kinematic viscosity, $v$, it is also useful to distinguish between the vorticity-diffusion stage, whereby velocity gradients are removed, and the species-diffusion stage, which removes scalar gradients (e.g. Batchelor 1959; Dimotakis 1986). On the other hand, successful descriptions and models of mixing provide us with tests of aspects of turbulent flow that are difficult to probe by other means-experimentally, numerically, or theoretically-at the high Reynolds numbers of interest here.

As a practical matter, fully-developed turbulent flow requires a Reynolds number,

$$
R e=\frac{U \delta}{v},
$$

that is high enough (e.g. G. I. Taylor, in Goldstein 1938, Ch. V; Batchelor 1953; Hinze 1975; and Monin \& Yaglom 1975). While, with some care, laminar flow can be observed at high Reynolds numbers, the converse is not true: turbulence cannot be sustained if the Reynolds number falls below some minimum value. In (1), the velocity, $U$, driving the turbulence and transverse extent of the flow, $\delta$, are to be 
taken as local values, as appropriate, i.e. $U=U(x)$ and $\delta=\delta(x)$, and therefore, also, $\operatorname{Re}=\operatorname{Re}(x)$. The choices for $U$ and $\delta$ will be illustrated in the context of the flows discussed below.

In the case of shear layers, for example, the characteristic velocity, $U$, will be taken as the (constant) free-stream velocity difference, i.e. $U_{s l}(x)=\Delta U=U_{1}-U_{2} \neq \mathrm{fn}(x)$, for a non-accelerating flow $(\mathrm{d} p / \mathrm{d} x=0)$, whereas the characteristic length, $\delta(x)$, will be taken as the local shear layer width, e.g. the visual thickness (Brown \& Roshko 1974), or, for chemically reacting shear layers, the $1 \%$ chemical-product profile thickness (Mungal \& Dimotakis 1984; Koochesfahani \& Dimotakis 1986, hereafter referred to as KD86). These are very close to each other and proportional to the downstream coordinate, $x$. Assuming constant fluid properties, i.e. $v=$ const., this yields a local Reynolds number for shear layers that increases linearly with the streamwise coordinate, i.e.

$$
R e_{s l}(x) \equiv \frac{\Delta U \delta_{s l}(x)}{v} \propto x .
$$

In the case of round, turbulent jets, the characteristic velocity can be taken as the local centreline velocity of the jet, i.e. $U(x)=U_{j}(x)=u_{c l}(x) \propto 1 / x$, while the local length scale is typically taken as the local jet diameter, i.e. $\delta(x)=\delta_{j}(x) \propto x$. This yields a local Reynolds number for jets that is a constant of the flow, i.e.

$$
R e_{j}(x) \equiv \frac{u_{j 0} d_{j}}{v} \simeq \frac{u_{c l}(x) \delta_{j}(x)}{v} \neq \mathrm{fn}(x) .
$$

This difference between shear layers and jets, for example, in the dependence of the local Reynolds number is interesting in the context of spatially developing flows and the evolution of the distribution of scales and turbulence spectra.

As the flow Reynolds number is increased from small values to values approaching some minimum Reynolds number for fully-developed turbulence, the flow is able to generate ever-increasing interfacial area between the mixing species, increasing mixing thereby and, in the case of chemically-reacting flow, chemical-product formation and heat release. Beyond this transition region, i.e. for $R e>R e_{\min }$, the Reynolds number dependence of the amount of mixed fluid can be expected, and found, to be weaker. While, for many flows, this transition is most conspicuous in terms of changes in the scalar field and mixing properties, hence its identification as the 'mixing transition', it also reveals itself through other measures, as will be discussed below.

\section{The mixing transition in various flows}

The mixing transition under consideration here is one that takes place beyond the well-documented transition from laminar to unsteady flow. The latter can often be understood in terms of hydrodynamic-stability arguments and, generally, reflects the inability of the flow to remain stable as the damping effects of viscosity are reduced with increasing Reynolds number. The mixing transition occurs at Reynolds numbers beyond those and represents a subsequent, often well-identified, further transition in the flow.

\subsection{Mixing transition in shear layers}

Konrad, in his 1976 thesis, notes that his gas-phase shear layers transition to threedimensionality at a particular value of the local Reynolds number, which he associates with the ability of the flow to sustain three-dimensional fluctuations: 
(a)
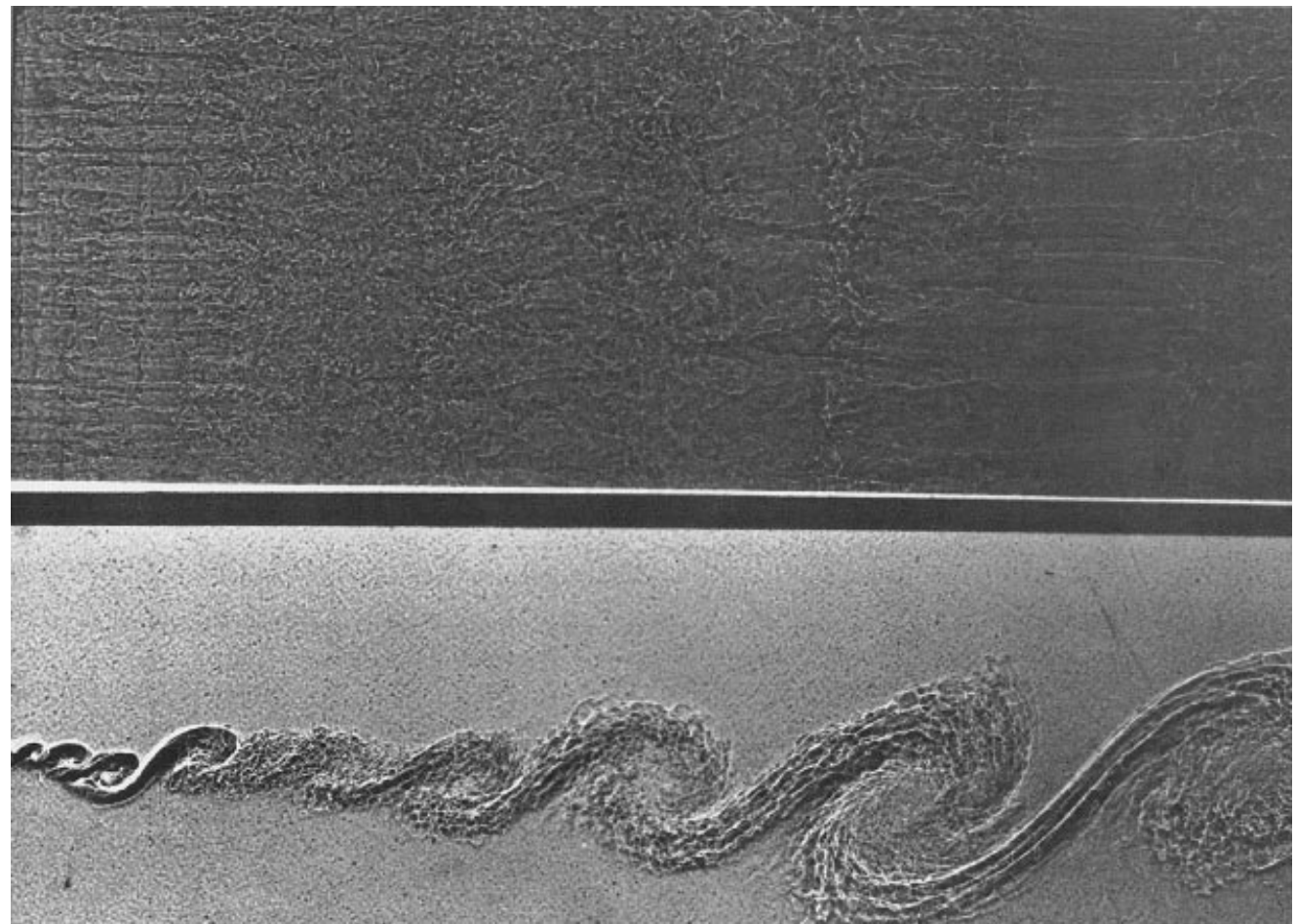

(b)

FIGURE 1. Plan- $(a)$ and side-view $(b)$ schlieren images of a gas-phase shear layer $\left(\mathrm{N}_{2}\right.$ over a density-matched mixture of $\mathrm{Ar}$ and $\mathrm{He}$ ). Note the rather abrupt transition in the early part of the flow (Konrad 1976, figure 2f).

This three-dimensionality corresponds in the photographs to the appearance of longitudinal (streamwise) lines and a 'dimpling' of the surface of the large structures (apparent on the upstream and downstream edges of the structure in the plan-view photographs). This phenomenon first appears at a Reynolds number $\left(\delta \Delta U / v_{\mathrm{N}_{2}}\right)$ of approximately $10^{4}$.

This transition is illustrated in the short-time exposure, simultaneous plan- and sideview schlieren image reproduced in figure 1 . As can be gleaned from this visualization, the transition is rather abrupt and results in a reduction of the steep, spanwise-aligned, scalar (index-of-refraction) gradients registered in the schlieren image $(a)$. The increase in disorganized three-dimensionality is evident in the spanwise image $(b)$.

The probability density function (p.d.f.) of the conserved scalar in the flow in the more well-mixed state in gas-phase $(S c \approx 1)$ turbulent shear layers noted by Konrad was measured using an aspirating probe (Brown \& Rebollo 1972) to estimate the local distribution of the high-speed free-stream fluid (mole) fraction values, averaged over the effective resolution-volume and time response of the aspirating probe.

Subsequent estimates of mixing and chemical product volume fraction in liquidphase shear layers by Breidenthal (1981), using a pH indicator, as well as estimates from probability-density functions (p.d.f.s) measured using laser-induced fluorescence techniques (KD86), confirmed this behaviour. A qualitative difference in the appearance of the scalar field is observed across this transition, which results in a more 
(a)

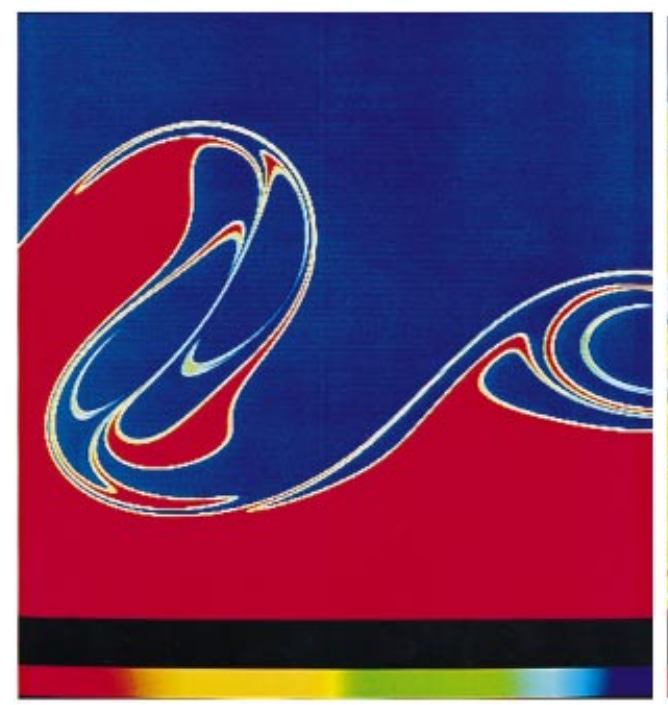

(b)

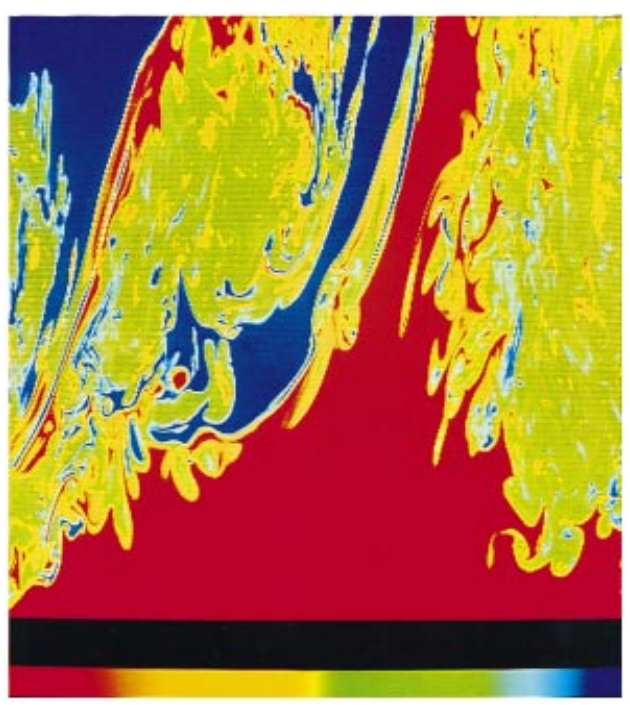

FIGURE 2. Laser-induced fluorescence streak images of the scalar field in a liquid-phase shear layer, for $R e \simeq 1.75 \times 10^{3}(a)$ and $R e \simeq 2.3 \times 10^{4}(b)$. From KD86, figures $7 b$ and 9, respectively.

well-mixed state, as illustrated in figure 2. The laser-induced-fluorescence image on the left $(a)$ recorded at a lower local Reynolds number $\left(R e \simeq 1.75 \times 10^{3}\right)$, represents a space-time $(y,-t)$ slice, at a fixed downstream location, $x$, in the midspan plane and indicates a near-cylindrical interfacial surface between the high-speed free-stream fluid (blue) and the low-speed free-stream fluid (red), with little mixing (KD86); 'near-cylindrical' because there is some three-dimensionality in the flow, as evidenced by the fact that not all regions are simply-connected (cf. 'islands' and 'lakes'), and as also surmised from the Konrad data (figure 1). The flow on the right $(b)$, at a local Reynolds number of $R e \simeq 2.3 \times 10^{4}$, is characterized by a much-higher mixing level. A large (space-time) area fraction is given to intermediate compositions (cf. mixture fraction colour bar: $0 \leqslant \xi \leqslant 1$ ).

As can be seen, the lower Reynolds number image indicates very high scalar gradients, at least for this liquid-phase (high-Sc) flow, with mixed fluid occupying a very small fraction of the volume. Conversely, at higher Reynolds numbers, the flow is characterized by lower scalar gradients, with a larger fraction of the flow occupied by intermediate composition (mixed) fluid. Interestingly, this scalar-gradient behaviour is opposite to what one would expect on the basis of a classical-spectrum vantage point (Oboukhov 1949; Corrsin 1951; Monin \& Yaglom 1975), wherein the highest wavenumbers (and scalar-gradient values) anticipated increase with increasing Reynolds number. On the other hand, Reynolds number values here are not high enough to warrant the invocation of such fully-developed turbulence theories.

Results from the two liquid-phase shear layer measurements are plotted in figure 3, which depicts the estimated chemical product thickness as a function of the local Reynolds number at the measuring station. A marked increase in the estimated chemical product can be seen to occur at $R e \approx 10^{4}$ (bottom scale). This increase is also associated with a change in the p.d.f. of the scalar fluctuations. In the pretransition region, the p.d.f. of the conserved scalar (high-speed-fluid fraction) in the flow is dominated by the near-delta-function free-stream contributions of the 


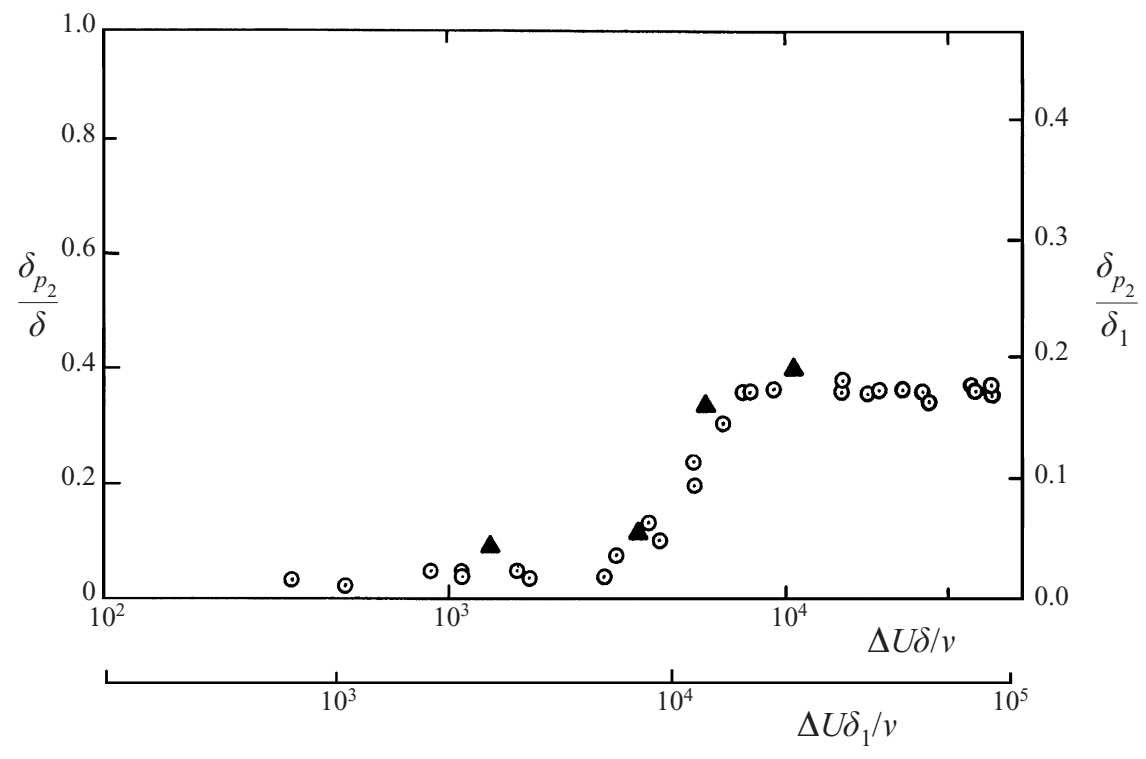

FIGURE 3. Reynolds number dependence of chemical product volume fraction, in a liquid-phase shear layer, in the vicinity of the mixing transition (KD86, figure 13). Local Reynolds number, as defined above (1), corresponds to lower scale. Absolute chemical-product values are overestimated in both experiments (see text).
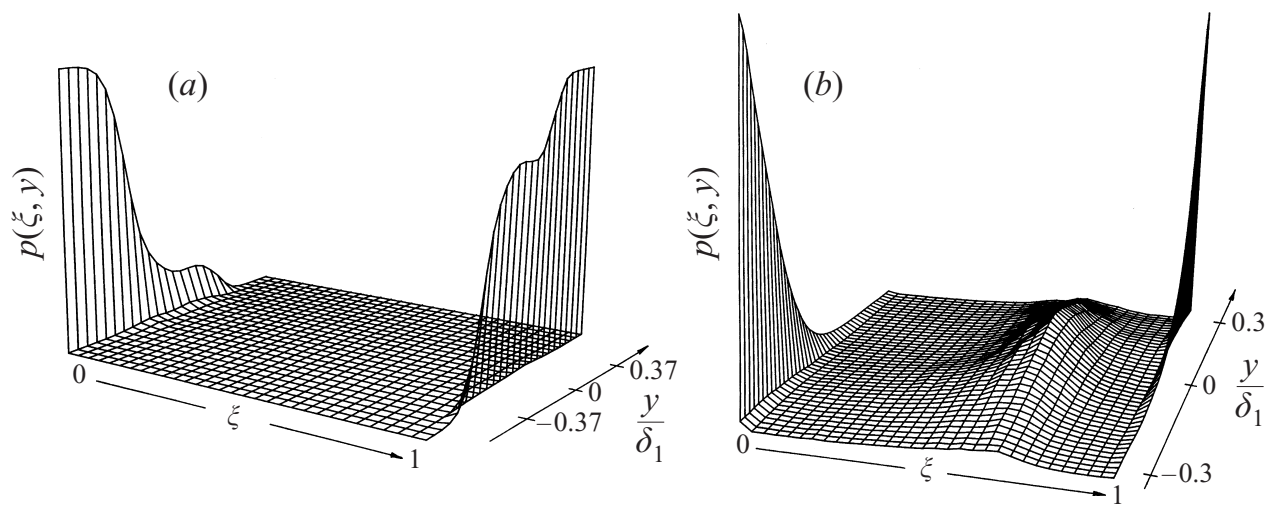

FIGURE 4. Estimated probability density function of the high-speed-free-stream fluid mole fraction, in the pre- $\left(R e \simeq 1.75 \times 10^{3}, a\right)$ and post-transition regime $\left(R e \simeq 2.3 \times 10^{4}, b\right)$. $\xi$ denotes mixed-fluid mole fraction (KD86, figures 8,10$)$.

unmixed (pure) fluid (KD86). For this flow, the composition of the mixed fluid across the layer develops a preferred value in the post-transition regime, that is well-correlated with the one inferred from the estimated overall entrainment ratio for the layer (Dimotakis 1986; KD86). In the course of the mixing transition, the p.d.f. evolves from one limit to the other (cf. KD86, § 5.4; and Masutani \& Bowman 1986). See figure 4. It is also characterized by a rather long 'memory' of the (typically, much larger) initial compositional asymmetry in the relative contributions of each of the free-stream fluids to the mixed fluid.

As noted in the discussions of these experiments (Breidenthal 1981, KD86), finiteresolution limitations in these liquid-phase experiments overestimated the absolute 
amount of chemical product by, roughly, a factor of two, as confirmed in chemicallyreacting experiments which measured the chemical product volume fraction directly (KD86). Nevertheless, the documented increase in the amount of mixing at the transition Reynolds number is qualitatively correct and was found to occur at the same Reynolds number in both gas- and liquid-phase shear layers (Bernal et al. 1980).

The transition to a more well-mixed state, in these experiments, was correlated with the appearance of streamwise vortices and the ensuing transition to threedimensionality of flow that is nominally two-dimensional in the initial/inflow region (Konrad 1976; Breidenthal 1981; KD86, Masutani \& Bowman 1986; Bernal et al. 1980; Bernal \& Roshko 1986). See also the discussion in the review paper by Roshko (1990). Corroborating evidence was also found in the numerical simulations of temporal shear layers by Moser \& Rogers (1991) that followed the developing flow to sufficiently high Reynolds numbers to document the beginning of this behaviour.

Jimenez, Martinez-Val \& Rebollo (1979) measured velocity fluctuations in a twodimensional shear layer and found a power-law regime in the energy spectrum, with an exponent close to $-\frac{5}{3}$, developing in the neighbourhood of the mixing transition. Subsequent investigations of the mixing transition by Huang \& Ho (1990) also associated the development of an, approximately, $\left(-\frac{5}{3}\right)$-slope spectral regime with the mixing transition, correlating it, however, with the number of pairings rather than with local values of the Reynolds number, as proposed previously by Dimotakis \& Brown (1976), who correlated it with Bradshaw's (1966) criterion in terms of initial momentum thicknesses, i.e. that we must have $x / \theta>10^{3} . \dagger$ Nevertheless, in these investigations, the Reynolds number in the vicinity of the mixing transition and the development of the $\approx-\frac{5}{3}$ spectrum regime was found to be in the range of $3 \times 10^{3}<\operatorname{Re}(x)<10^{4}$, in accord with the range documented in figure 3 .

Similar conclusions may be drawn from the work by Pawlak \& Armi (1998), on spatially accelerating, stratified shear layers. This flow is important because vorticity (circulation) is generated baroclinically, as opposed to canonical shear layers for which $\Delta U=U_{1}-U_{2}$ is constant with a fixed circulation per unit length. As the authors note, this flow also provides a further illustration of differences 'between spatial and temporal flow' (cf. Thorpe 1971, 1973). Despite differences in the dynamics, the authors note the late-stage evolution of their flows into a more-developed state. The highest local Reynolds number in those experiments may be estimated to be just below $10^{4}$, or so, but the flow exhibits the beginnings of a qualitative transition at the final downstream station, where $\operatorname{Re}\left(x_{\text {final }}\right) \approx 5 \times 10^{3}$.

The investigations cited above support the notion of a turbulent transition in shear layers at a local Reynolds number, $R e \approx 1-2 \times 10^{4}$. This number should be regarded as imposing a necessary, but not sufficient condition, however. As discussed in Breidenthal $(1981, \S 6)$, additional requirements involving the flow inflow conditions can be argued for. By way of example, one can conceive of initially-laminar boundary layers peeling off the splitter-plate trailing edge to form a shear layer that could be thick enough for the local Reynolds number to be sufficiently high from the outset. Hydrodynamic-stability considerations, however, would suggest that such a flow would require some distance downstream for the Kelvin-Helmholtz instability to develop that would be scaled by the initial momentum thickness (and wake profiles).

$\dagger$ See Slessor, Bond \& Dimotakis (1998) for a recent discussion on this topic and experiments on the effects of initial conditions on far-field shear-layer behaviour and mixing, indicating that the state of fully-developed turbulence at high Reynolds number can depend on initial/inflow conditions. 
As the Kelvin-Helmholtz instability would have to develop first, an additional distance downstream can then be anticipated for the mixing transition to occur, corresponding to a higher local Reynolds number yet.

The behaviour of canonical shear layers is not only important in its own right, but also because local shear-layer regions can be found in other, as well as morecomplicated, flow fields. As a consequence, transitional behaviour observed in other flows may be traceable to transitional behaviour in local shear-layer regions in those flows, as will be discussed below.

\subsection{Mixing transition in jets}

The transition to a more well-mixed state in turbulent jets is less conspicuous than in shear layers. Turbulent-jet flow being three-dimensional, even at low Reynolds numbers, such a transition is not correlated with a transition to three-dimensionality, as it can be in shear layers, for example. Nevertheless, as we shall discuss, experiments indicate a qualitative difference in the behaviour of the scalar field, for values of the Reynolds number that are lower than $R e_{\min } \approx 10^{4}$ and values comparable to, or higher than, that, as well as in various other quantitative measures. $\uparrow$ This change in behaviour can be gleaned from both non-reacting and chemically-reacting jets.

Experiments on scalar-mixing behaviour in non-reacting jets indicate a qualitative transition in the turbulence and turbulent-mixing behaviour for Reynolds numbers in the vicinity of $10^{4}$. An illustration of this transition can be found in the laser-inducedfluorescence images in figure 5 , of the jet-fluid concentration in the plane of symmetry of liquid-phase turbulent jets (Dimotakis, Miake-Lye \& Papantoniou 1983).

Unmixed reservoir fluid (black) can be seen throughout the turbulent region and, in particular, all the way to the jet axis in the lower Reynolds number image $(a)$ at $R e \simeq 2.5 \times 10^{3}$. The imaged field spans $0<z / d_{j}<35$, where $d_{j}$ is the jet-nozzle diameter and, here, $z$ is the streamwise coordinate. This is not the case in the higher Reynolds number $(b)$ image at $R e \simeq 10^{4}$ (imaged field spans $0<z / d_{j}<200$ ), in which jet fluid of varying concentrations can be seen to be more volume-filling within the turbulent region.

Seitzman et al. (1990) investigated the outer entrainment and mixing region, using laser-induced-fluorescence images of $\mathrm{OH}$ radicals in a $\mathrm{H}_{2}$-air turbulent diffusion flame. A qualitative evolution in the complexity of the thin burning regions can be seen as the Reynolds number was increased from $2.3 \times 10^{3}$ to $4.95 \times 10^{4}$ (cf. their figure 3). In these experiments, this evolution is also influenced by decreasing buoyancy and the decreasing relative importance of baroclinic vorticity generation, as the Reynolds number was increased, and is, therefore, not entirely attributable to Reynolds number effects.

Similar behaviour is reflected in the measurements of the r.m.s. of the scalar (jet fluid concentration) fluctuations on the axis, in the far field of gas- and liquid-phase jets, as a function of jet Reynolds number (Dowling \& Dimotakis 1990; Miller 1991; and Miller \& Dimotakis 1991). The data, in the form of the normalized scalar fluctuation variance, are plotted in figure 6 (Miller 1991, figure 7.2). The liquid-phase data exhibit a decrease in the fluctuation level with Reynolds number, with a rather less sensitive dependence for Reynolds numbers higher than $R e \approx 2 \times 10^{4}$ or so. Noting that lower fluctuation levels correspond to more homogeneous mixing, i.e. a p.d.f. of concentration values that are more tightly clustered around the local

$\dagger$ In the discussion of jet Reynolds numbers, no distinction will be made between the jet-nozzle flow Reynolds number and local far-field Reynolds number, the two being essentially equal for the purposes of the present discussion $(2 b)$. 
(a)

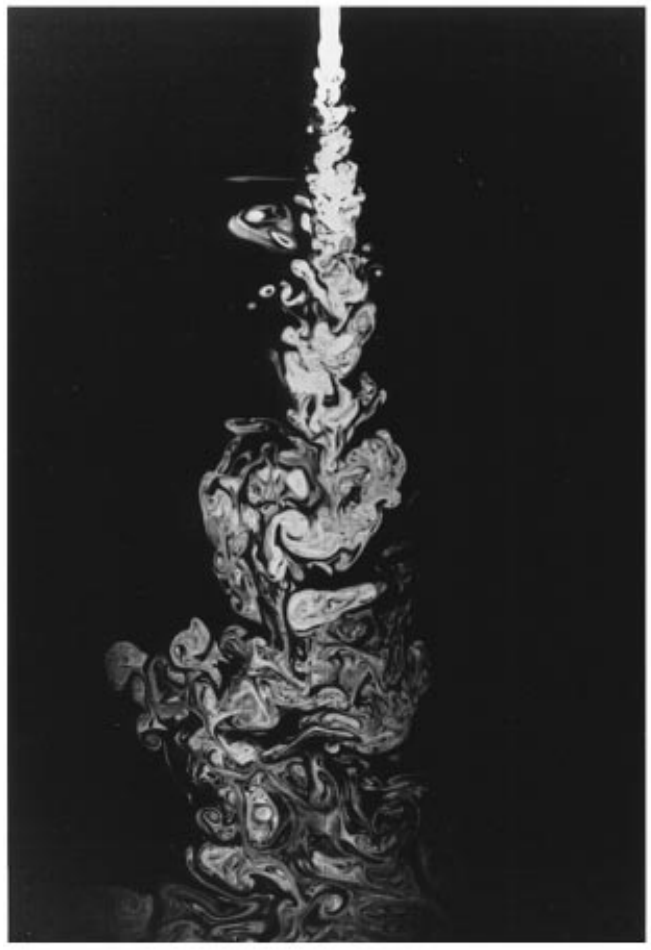

(b)

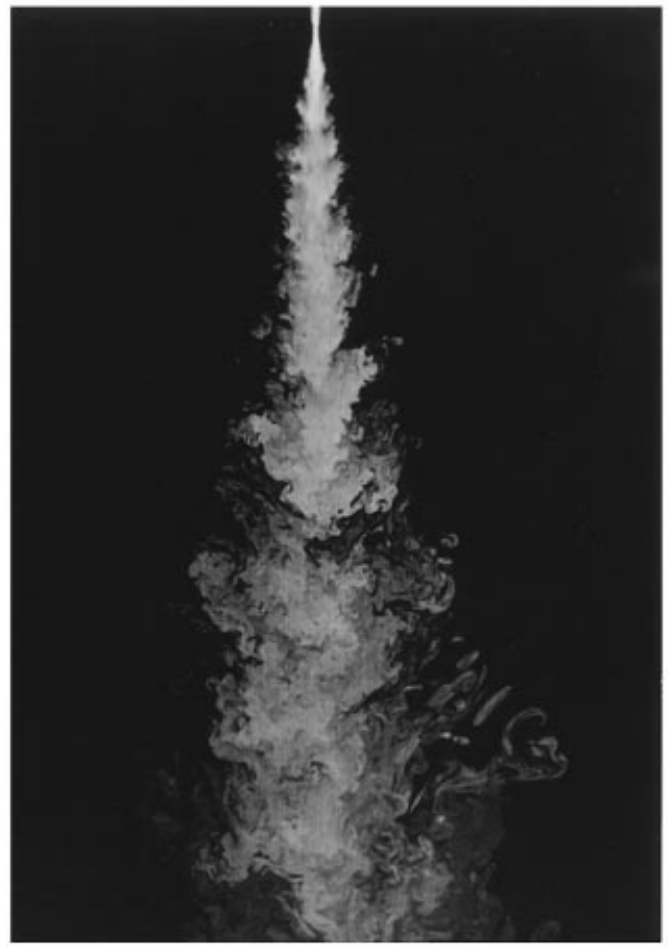

FIGURE 5. Jet-fluid concentration in the plane of symmetry of a round turbulent jet. (a) $R e \simeq 2.5 \times 10^{3}$ $\left(0<z / d_{j}<35\right)$. (b) $R e \simeq 10^{4}\left(0<z / d_{j}<200\right)$. Data from Dimotakis et al. (1983, figures 5 and 9$)$.

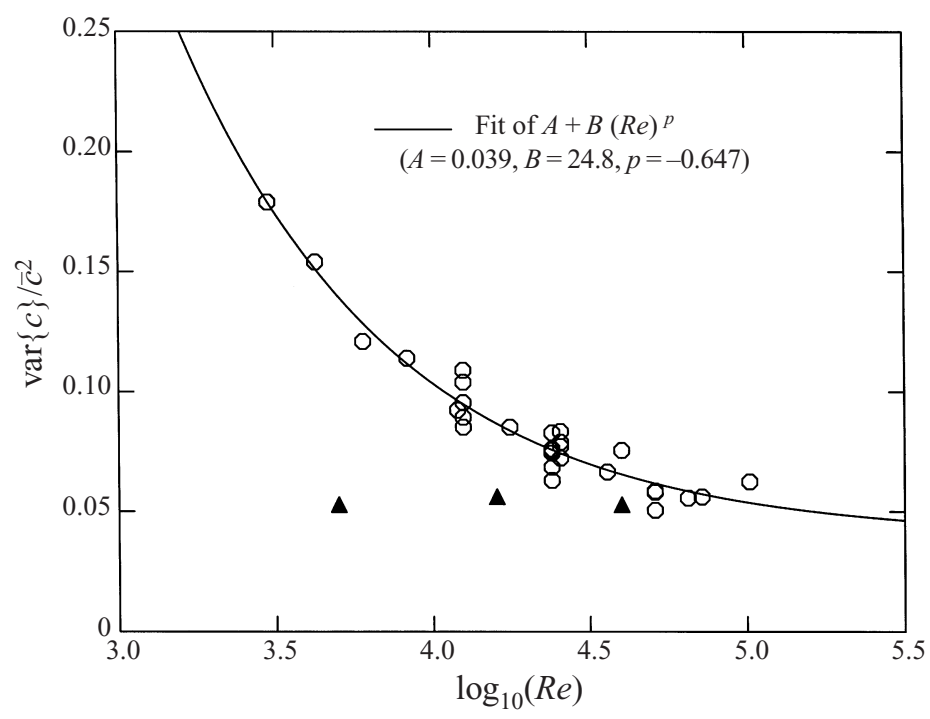

FIGURE 6. Normalized jet-fluid-concentration variance on the axis of a turbulent jet, as a function of jet Reynolds number (Miller 1991, figure 7.2). Circles: liquid-phase jets (Miller 1991); triangles: gas-phase jets (Dowling \& Dimotakis 1990). 
(a)

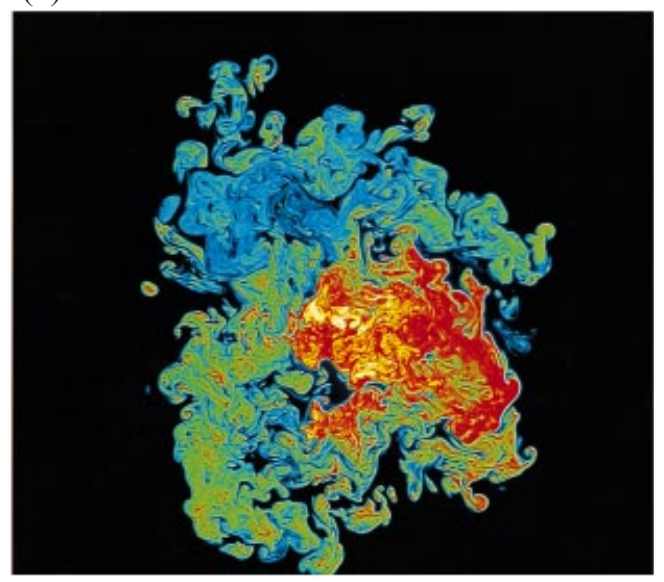

(b)

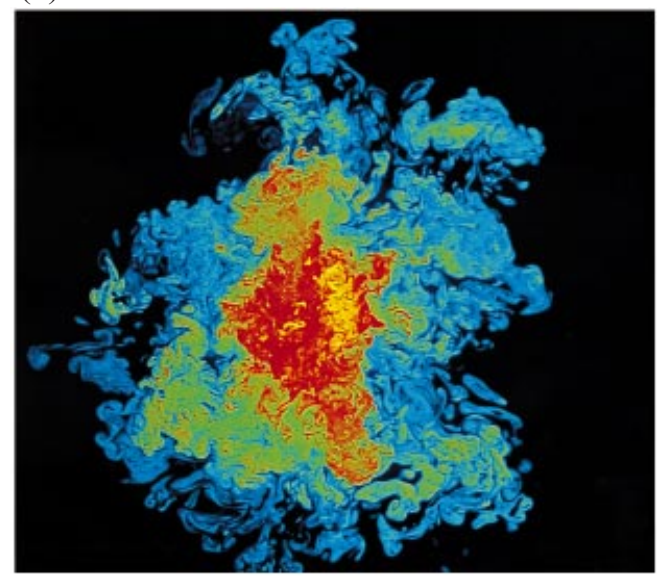

FiguRE 7. Laser-induced fluorescence far-field $\left(z / d_{j}=275\right)$ jet-fluid concentration field in a liquidphase turbulent jet, at $R e \simeq 4.5 \times 10^{3}(a)$ and $18 \times 10^{3}(b)$, in a plane normal to the jet axis (CD96, figures 3 and 5). Colour map codes jet-fluid concentration.

mean, we see that, at least for the case of a liquid-phase jet, the flow transitions to a more well-mixed state as the Reynolds number is increased, as in the shear layer, even though in a more gradual manner (cf. figure 3). A much weaker, if any, Reynolds number dependence of the normalized scalar variance can be seen for the gas-phase-jet data. This difference in behaviour must be attributed to a Schmidt number effect. Specifically, for $S c \approx 1$, the greater molecular species diffusion bridges recently entrained reservoir-fluid filaments, better homogenizing the scalar field even at lower Reynolds numbers. For the liquid-phase jets, $S c \approx 10^{3}$, improved mixing requires the enhancement in interfacial surface-to-volume ratio (smaller distances between isoscalars) associated with higher Reynolds numbers.

In experiments by Liepmann \& Gharib (1992), in the near field of turbulent jets, the number of azimuthal nodes in vortex structures becomes difficult to identify beyond a certain Reynolds number, where the flow transitions to a much more chaotic state. The jet near-field region is dominated by a near-axisymmetric shear-layer region, so that such a transition may be rightly considered as a shear-layer transition in its origins. Nevertheless, it persists beyond the jet-core region $\left(z / d_{j} \lesssim 5-6\right)$ and may be regarded as a transition in the near field of the jet. The authors correlate it with a laminar-turbulent transition in the jet-nozzle boundary layers. It is also interesting, however, that it occurs at a jet Reynolds number close to $10^{4}$.

Evidence for a mixing transition in jets can be seen in the more-recent liquidphase $\left(S_{c} \simeq 2.0 \times 10^{3}\right)$ turbulent-jet measurements, at three Reynolds numbers $(R e \simeq$ $4.5 \times 10^{3}, 9.0 \times 10^{3}$, and $18 \times 10^{3}$ ), in the transverse, far-field, laser-induced-fluorescence cuts of the jet-fluid concentration field (Catrakis \& Dimotakis 1996, hereafter referred to as CD96). A pair of such images is shown in figure 7. These data span the full transverse extent of the jet-fluid-concentration field, at the $z / d_{j}=275$ downstream measurement location.

As with the shear-layer data (figure 2), we see (figures 5 and 7) unmixedness and steeper scalar gradients associated with the pre-transitional state. The increase in mixing in the post-transitional flow anneals steep scalar gradients, driving the scalar field to a more homogenized state. This change in behaviour is also registered in the 


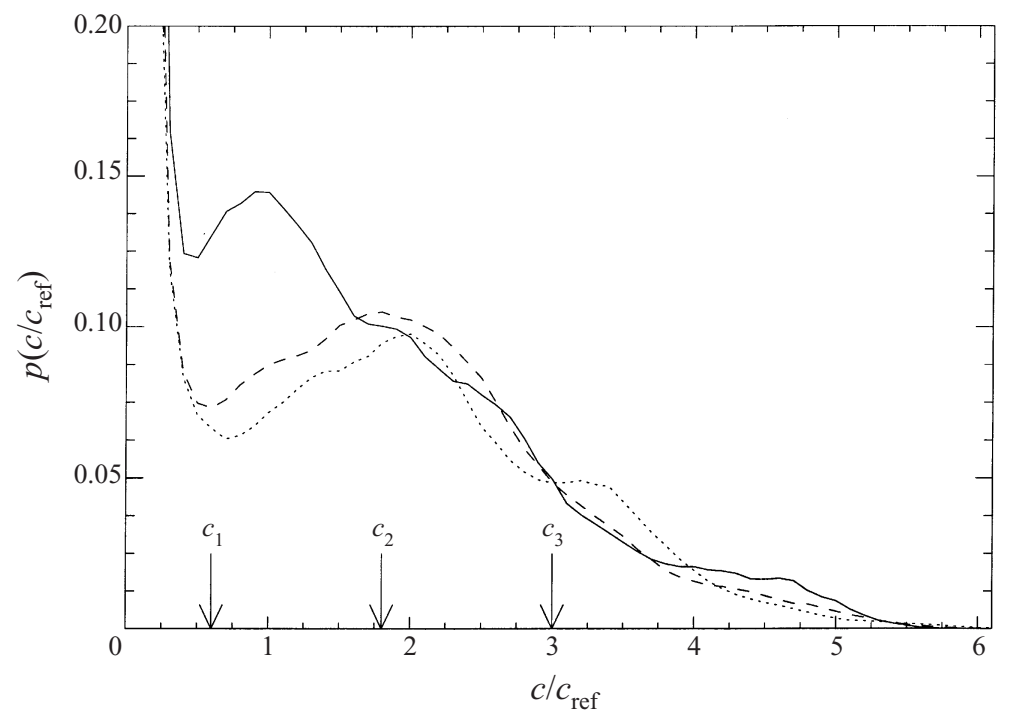

FIGURE 8. Jet-fluid concentration p.d.f. in the far field $\left(z / d_{j}=275\right)$ of a turbulent jet. $R e \simeq 4.5 \times 10^{3}$ (dotted line); $R e \simeq 9.0 \times 10^{3}$ (dashed line); $R e \simeq 18 \times 10^{3}$ (solid line). Three scalar-threshold values, $c_{1}, c_{2}$, and $c_{3}$, are also indicated (CD96, figure 8).

p.d.f. of the scalar field, as illustrated in figure 8. As can be seen, the scalar (jet-fluid concentration) field has a preferred value for pre-transitional Reynolds numbers, i.e. $c / c_{\text {ref }} \approx 2$, where $c_{\text {ref }} \simeq c_{0} / 200$ with $c_{0}$ the initial (jet-plenum) concentration, whereas the lower probability of compositions between $c / c_{\text {ref }} \approx 2$ and the p.d.f. 'bucket' representing unmixed reservoir fluid $\left(c / c_{\text {ref }} \rightarrow 0\right)$ is largely filled at $R e \simeq 18 \times 10^{3}$ (cf. CD96, figure 8 and related discussion). One could speculate that the jet-fluid concentration p.d.f., $p\left(c / c_{\text {ref }}\right)$, will become monotonically decreasing, i.e. no bucket, at yet higher Reynolds numbers.

While this may be a minor point, we note that transition Reynolds numbers for jets seem to be twice as large as for shear layers. On the one hand, the two flows are sufficiently different to admit differences in their behaviour of a factor of 2 , or so, in Reynolds number. On the other, however, if the characteristic large scale $\delta(x)$ chosen for the local Reynolds number definition of a jet is the local radius, as would be appropriate if the length scale in the general case is defined as the transverse spatial extent across which the shear is sustained, then the transition Reynolds number for jets becomes very close to those for shear layers.

The Reynolds number dependence of turbulent mixing and chemical product formation in turbulent jets was investigated in gas-phase jets (Gilbrech 1991; Gilbrech $\&$ Dimotakis 1992). In this context, the turbulent-diffusion flame length, $L_{f}$, is important, marking the distance from the nozzle required to mix and burn the reactant carried by the jet, mixed to the composition dictated by the stoichiometric mixture ratio, on a molecular scale. If the stoichiometry of the jet/reservoir reactants and jet entrainment are held constant, and for fast chemical kinetics (high Damköhler number limit), the flame-length dependence on the various flow parameters provides us with a measure of the dependence of mixing on those parameters. Decreasing flame lengths, for example, imply faster (better) mixing.

The dependence of the flame length on the stoichiometry of the jet-/reservoir-fluid chemical system must first be factored in the analysis. In particular, for a momentum- 


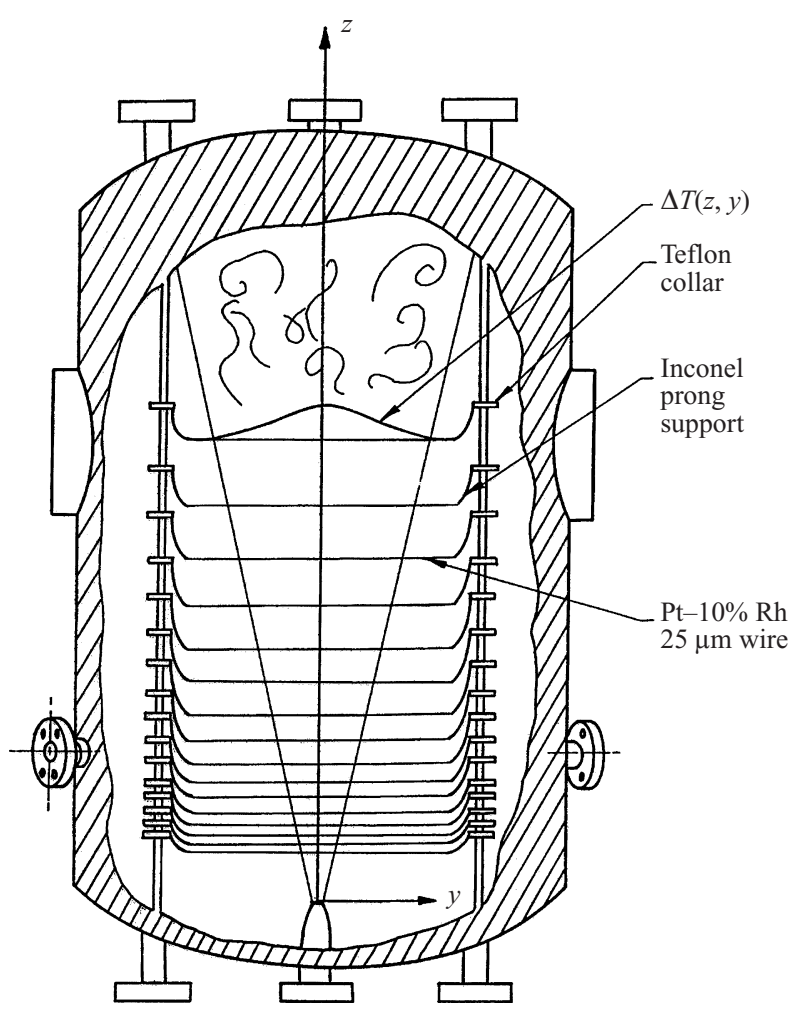

FIGURE 9. Turbulent-jet diffusion flame combustion vessel schematic, indicating the jet and logarithmically-spaced temperature-sensing wire array (Gilbrech 1991, figure 2.4).

dominated turbulent jet diffusion flame, the flame length is linearly dependent on the (mass) stoichiometric mixture ratio (e.g. Hottel 1953), i.e.

$$
\frac{L_{f}}{d^{*}} \simeq A \phi_{m}+B, \quad \text { where } \quad d^{*} \equiv \frac{2 \dot{m}_{0}}{\left(\pi \rho_{\infty} J_{0}\right)^{1 / 2}}
$$

is the jet source diameter, with $\dot{m}_{0}$ the mass flux and $J_{0}$ the momentum flux of the jet discharge (Dimotakis 1984; Dahm \& Dimotakis 1987), and $\phi_{m}$ is the mass of reservoir fluid required to completely consume (react with) a unit mass of jet fluid (Broadwell 1982). The measurements may then be regarded as investigations of the behaviour of the stoichiometric coefficient, $A$, and normalized virtual origin (intercept), $B$, and their dependence, in turn, on the flow parameters.

In these experiments, long platinum wires were stretched across the turbulent diffusion flame and spaced in equal logarithmic increments along the jet axis. These permitted the line-integral of the temperature rise, $\Delta T(z, y)$, due to heat released in the chemical reaction, to be measured along the $y$-coordinate (transverse to the jet axis), as a function of the downstream coordinate. See figure 9.

The experiments utilized the $\mathrm{F}_{2}+\mathrm{NO}$ chemical reaction, with $\mathrm{F}_{2}$ diluted in $\mathrm{N}_{2}$ forming the jet fluid, and $\mathrm{NO}$ diluted in $\mathrm{N}_{2}$ forming the quiescent reservoir fluid. With this chemical system, an adiabatic flame temperature rise, $\Delta T_{f}$, as low as $7 \mathrm{~K}$ was realized, with the reaction still in the fast-kinetic regime. Such low values were dictated by the results of a separate investigation that assessed the effects of buoyancy and ascertained that the measurements were realized in the momentum-dominated 


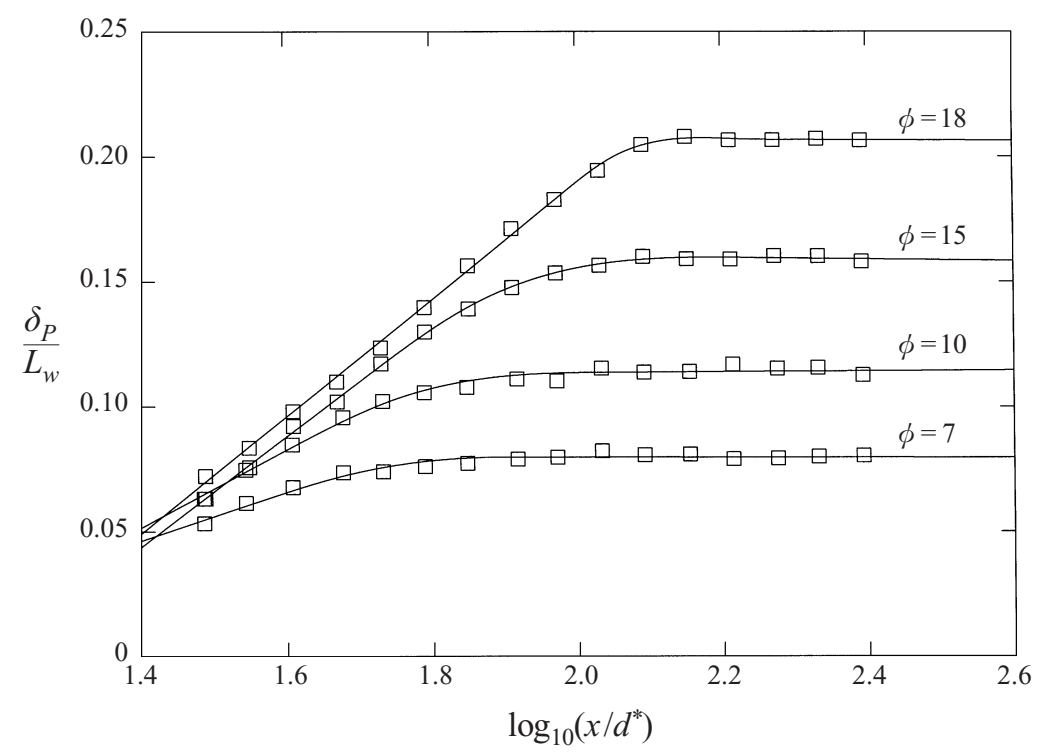

FIGURE 10. Product thickness normalized by wire length vs. $\log _{10}\left(z / d^{*}\right)$, for several stoichiometric mixture ratios (Gilbrech \& Dimotakis 1992, figure 5).

regime for this heat-releasing flow. The Reynolds number was varied by varying the pressure in the combustion vessel.

If the temperature rise, $\Delta T(z, y)$, in the chemically-reacting jet is normalized by $\Delta T_{f}$, the adiabatic flame temperature rise for the reaction, the line integral across the jet axis can be used to form a product thickness, $\delta_{P}(z)$, analogous to the one defined for shear layers, i.e.

$$
\delta_{P}(z) \equiv \int_{-\infty}^{\infty} \frac{\Delta T(z, y)}{\Delta T_{f}} \mathrm{~d} y
$$

(cf. Bilger 1980, §3.1.3; Kuo 1986, §1.9; and Dimotakis 1991, equations 41 and discussion following).

Sample data are depicted in figure 10, for a range of values of the (mass) stoichiometric mixture ratio, $\phi_{m}$. The data plot the product thickness $\delta_{P}$, normalized with the length, $L_{w}$, of the platinum resistance wire used to measure the line integral, versus the logarithm of $z / d^{*}$, where $d^{*}$ is the jet source diameter (3).

We note that well upstream of the flame length, i.e. for $z \ll L_{f}$, the entrained reactant is consumed on, or just inside, the boundary of the turbulent region. There, the turbulent fluid is jet-fluid-reactant rich and it need comprise only a small fraction of the mixed fluid to consume the entrained reservoir-fluid reactant. The diffusion/reaction process then takes place in a thin peripheral reaction zone, at $y= \pm R_{r}(z)$, whose ensemble-averaged radius, $R_{r}(z)$, is proportional to the streamwise coordinate, $z$. This picture is corroborated by the $\mathrm{OH}$ images obtained by a number of investigators in $\mathrm{H}_{2}$-air jet flames (cf. Seitzman et al. 1990 and Namazian \& Kelly 1988 , for example). As a consequence, the line integral of the time-averaged temperature rise across the turbulent region increases as the chemical reaction releases heat in the thin reaction zones at the edges of the turbulent region.

It was conjectured that the radial integral of the temperature rise, at a given station, 
$z$, increases in proportion to the entrainment velocity at that station, $u_{e}\left[R_{r}(z)\right]$, i.e.

$$
\frac{\mathrm{d}}{\mathrm{d} z} \int_{0}^{\infty} \Delta T(z, r) \mathrm{d} r \propto u_{e}\left[R_{r}(z)\right] \Delta T_{f},
$$

or, for a momentum-driven turbulent jet,

$$
\mathrm{d} \int_{-\infty}^{\infty} \frac{\Delta T(z, y)}{\Delta T_{f}} \mathrm{~d} y \propto \frac{\mathrm{d} z}{2 \pi R_{r}(z)} \propto \frac{\mathrm{d} z}{z} .
$$

Integrating this relation and scaling with the flame length $L_{f}$, we have

$$
\frac{\delta_{P}\left(z \ll L_{f}\right)}{L_{f}} \simeq a \log \left(\frac{z}{L_{f}}\right)+b .
$$

This dependence of the line integral on $z$ suggested the logarithmic wire spacing used in the experiment and was used in the analytical form of the fit for the line-integrated, time-averaged, temperature-rise data (cf. figure 10).

Beyond the end of the flame region, i.e. for $z>L_{f}$, no further heat is released and, in the absence of buoyancy effects, the temperature excess becomes a passively-convected scalar with a self-similar profile. In that case,

$$
\Delta T(z, y) \simeq \Delta T(z, 0) f\left(\frac{y}{z}\right) \propto \frac{1}{z} f\left(\frac{y}{z}\right)
$$

and the product thickness line integral becomes independent of the downstream coordinate, $z$, i.e.

$$
\delta_{P}\left(z>L_{f}\right) \propto \int_{-\infty}^{\infty} \frac{1}{z} f\left(\frac{y}{z}\right) \mathrm{d} y \neq \mathrm{fn}(z) .
$$

As can be seen in figure 10, the experimental results confirm the conjecture for $z / L_{f} \ll 1$. They are also consistent with the anticipated conserved-scalar behaviour of the temperature rise for $z / L_{f}>1$, i.e. a product thickness that asymptotes to a constant value.

Such data allow us to estimate the flame length, $L_{f}$. In particular, one can accept an operational definition of $L_{f}$ as the location where the product thickness line integral (4) has attained $99 \%$ of its asymptotic value, as one does for a boundary-layer velocity profile, for example.

Figure 11 plots the stoichiometric coefficient, $A$, in the flame length (3), i.e. the slope of the flame length vs. the stoichiometric mixture ratio $\phi_{m}$ (Gilbrech 1991, figure 4.8). This may be regarded as the additional length, in units of the jet source diameter, $d^{*}$, required to entrain, mix, and react with a unit increase in the stoichiometric ratio of the jet-/reservoir-fluid chemical system (3), i.e.

$$
A \equiv \frac{\mathrm{d}}{\mathrm{d} \phi_{m}}\left(\frac{L_{f}}{d^{*}}\right) .
$$

In the fast-kinetic regime, as was the case in these experiments, this quantity is a useful measure of mixing. It separates the self-similar, far-field behaviour from that of the virtual origin in the overall mixing process.

The data in figure 11 indicate that mixing in the far field of a turbulent jet improves relatively rapidly with increasing Reynolds number. Specifically, $A$ decreases until a Reynolds number of, roughly, $2 \times 10^{4}$, with a much weaker dependence on Reynolds number, if any, beyond that. These data are in accord with the non-reacting, liquidphase data in figure 6, which also indicate improved mixing up to Reynolds numbers 


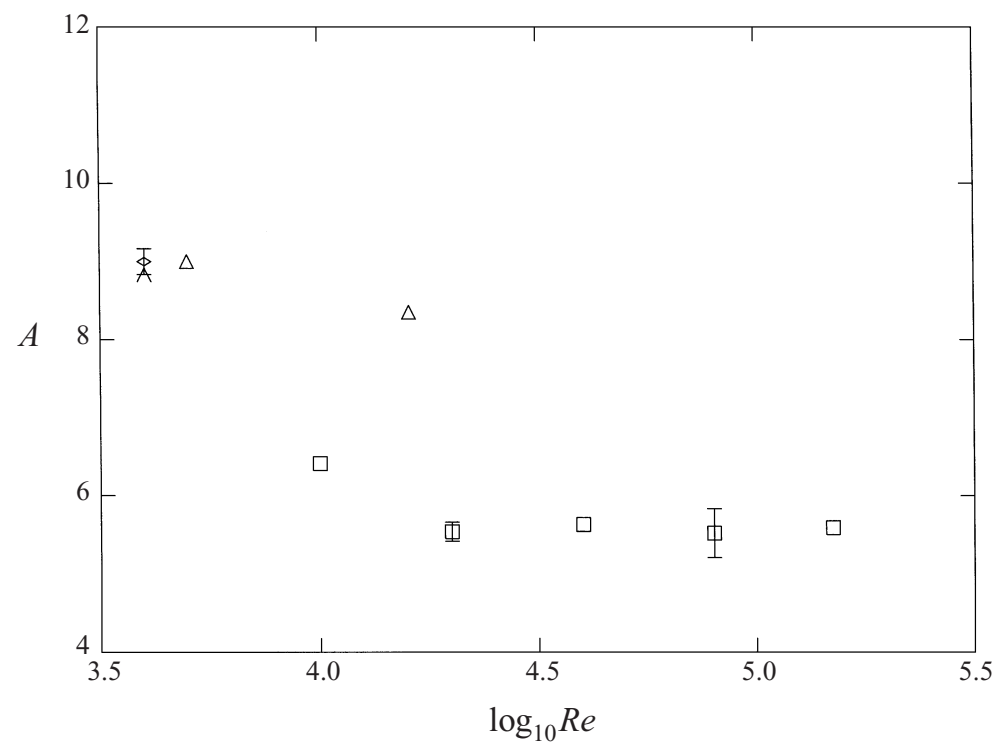

FIGURE 11. Flame length stoichiometric coefficient $A$ (3). Squares: gas-phase chemically-reacting jets (Gilbrech 1991); diamond: laser-induced fluorescence, liquid-phase jets (Dahm et al. 1984); Lambda: pH-indicator, liquid-phase jets (Hottel 1952; Weddel 1941); triangles: flame length data inferred from gas-phase, non-reacting jets (Dowling 1988, see text).

of $2 \times 10^{4}$, or so, with a weaker dependence beyond that. The latter data, however, do not permit the separation of the far-field and virtual-origin contributions to the overall mixing process, as do the chemically reacting jet data. We should also note that the near- and intermediate-field behaviour, which contributes to the virtual origin of the mixing process and the resulting flame length, does not exhibit the same Reynolds number dependence (Gilbrech 1991).

A potential difficulty should be noted between the inferred behaviour based on nonreacting, gas-phase jet data (figure 11: Dowling 1988, triangles), and the chemicallyreacting, gas-phase data (Gilbrech 1991, squares). The values estimated from the non-reacting gas-phase data were derived assuming certain similarity properties of the concentration p.d.f. and the value of the virtual origin of the mixing process (cf. discussion in Dowling 1988, §5.4, and Miller \& Dimotakis 1991, Appendix B). Partly as a consequence, as also noted in the comparison between the data in figures 6 and 11, it is not possible to separate the contribution to the flame length of the (rather large) mixing-process virtual origin, and its dependence on Reynolds number (Gilbrech 1991), from the Reynolds number dependence of the far-field mixing process, i.e. of the flame-length stoichiometric coefficient, $A$.

Similar behaviour was documented in experiments on lifted-flame behaviour by Hammer (1993), who notes a change in the scaled lift-off height of turbulent jet flames at a jet Reynolds number $R e \approx 1.8 \times 10^{4}$, beyond which the Reynolds number dependence is weaker. See data in his figure 3.8, and discussion following.

\subsection{Mixing transition in other flows}

The observations of mixing transitions in shear layers and jets suggest that a minimum Reynolds number may be required for turbulence to develop into a more wellmixed state in these flows. Specifically, we must have $R e>R e_{\min }$, with $R e_{\min }$ in the neighbourhood of $1 \times 10^{4}$ to $2 \times 10^{4}$, for fully-developed turbulent flow. This value 


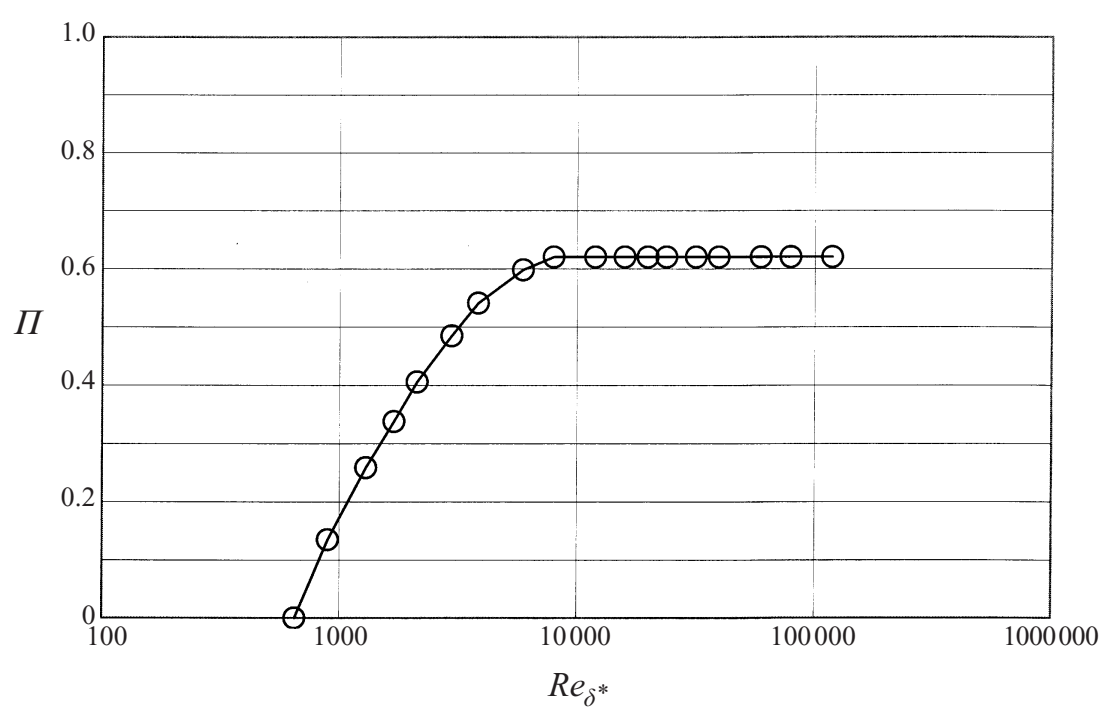

FIGURE 12 . Turbulent boundary-layer $\Pi$ parameter vs. $R e_{\delta^{*}}$. Skin-friction-law fit data (Collins et al. 1978, table 4).

does not appear to be peculiar to the far-field behaviour itself in turbulent jets and free-shear layers, two flows that are already substantially different by any of a number of different measures. As it turns out, other flows also exhibit similar transitions at comparable values of the Reynolds number, as we shall discuss below.

Pipe flow transitions out of its slug/puff regime to a less intermittent, fullydeveloped turbulent state over a range of Reynolds numbers that depends on the entrance conditions. This sensitivity to initial conditions diminishes, however, at a Reynolds number in the vicinity of $10^{4}$ (Wygnanski \& Champagne 1973).

Coles's (1956) turbulent boundary-layer wake parameter, $\Pi$, that scales the outer flow region of a turbulent boundary layer is found to increase with Reynolds number for a zero-pressure-gradient boundary layer, attaining an asymptotic value of $\Pi=0.620$ at a Reynolds number of $R e=R e_{\delta^{*}} \equiv U_{\infty} \delta^{*} / v \simeq 0.8 \times 10^{4}$, based on the boundary-layer displacement thickness (figure 12). Parenthetically, a turbulent boundary layer may be regarded as characterized by three transverse scales, whose separation increases with increasing Reynolds number: the inner, viscous sub-layer; the intermediate, log-law layer; and the outer, wake region layer. The integral displacement thickness was used here as the appropriate measure of the transverse extent of the flow and the length scale in the definition of the local Reynolds number. See Coles (1968) and Collins, Coles \& Hicks (1978) for a discussion, and their table 4 and figure 6 for a compilation of low-speed turbulent boundary-layer flow data.

In his review of bluff-body flows, Roshko (1993) documents several Reynolds number regimes, indicated by the base pressure of a circular cylinder. The (negative) base pressure increases in the range $0.3 \times 10^{4}<R e_{d} \equiv U_{\infty} d_{\text {cyl }} / v<2 \times 10^{4}$ (cf. Roshko 1993, figure 1). He attributes this behaviour to a transition in the separating shear layers, analogously to the documented near-field behaviour in jets. A similar conclusion may be reached on the basis of the fluctuating (sectional) lift coefficient (figure 13) that exhibits a weaker Reynolds number dependence for cylinder Reynolds numbers, $R e_{d} \gtrsim 10^{4}$ (Norberg \& Sundén 1987). 


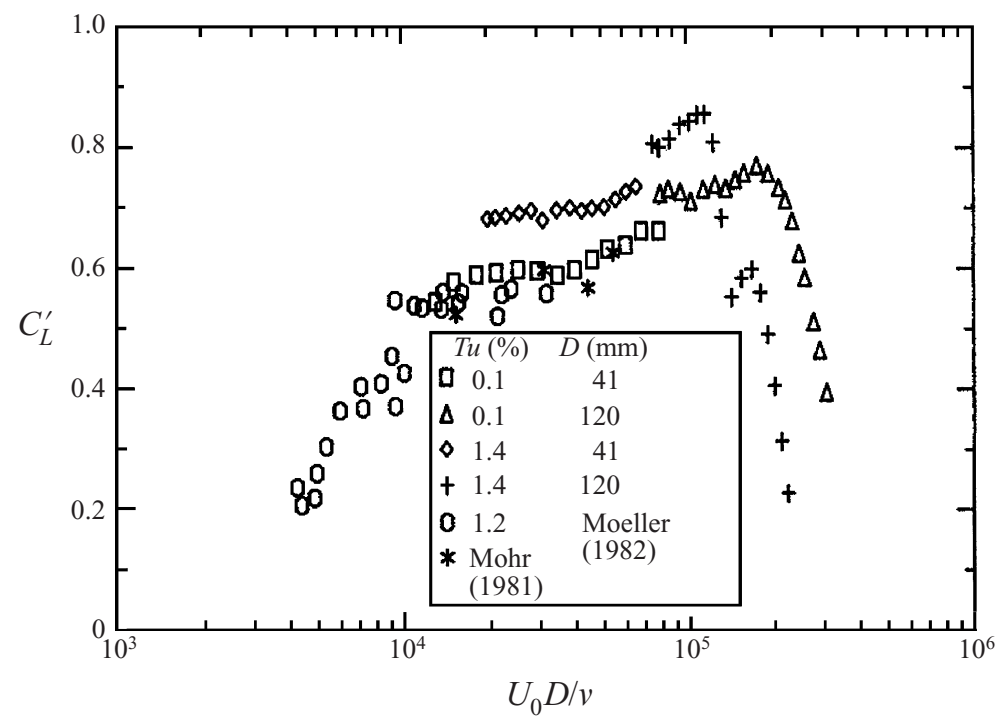

FIGURE 13. Fluctuating (r.m.s.) lift coefficient dependence on cylinder Reynolds number and free-stream turbulence level (Norberg \& Sundén 1987, figure 11).

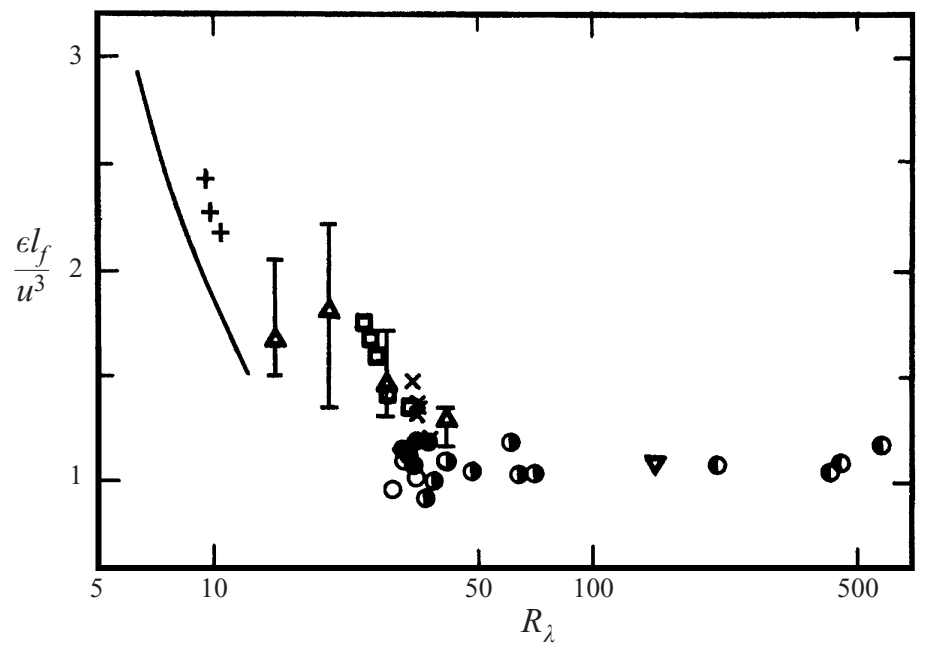

FIGURE 14. Scaled turbulent kinetic energy dissipation (10) vs. Taylor Reynolds number (Sreenivasan 1984, figure 1).

Measurements of the scaled turbulent kinetic energy dissipation rate, per unit mass, i.e.

$$
\alpha=\frac{\varepsilon l}{u^{\prime 3}},
$$

in flow behind square grids, where $\varepsilon$ is the kinetic energy dissipation per unit mass, $l$ is the longitudinal (integral) length scale, and $u^{\prime}$ is the r.m.s. velocity fluctuation level, show that $\alpha$ decreases relatively rapidly with increasing Taylor Reynolds number,

$$
\operatorname{Re}_{T} \equiv \frac{u^{\prime} \lambda_{T}}{v}
$$




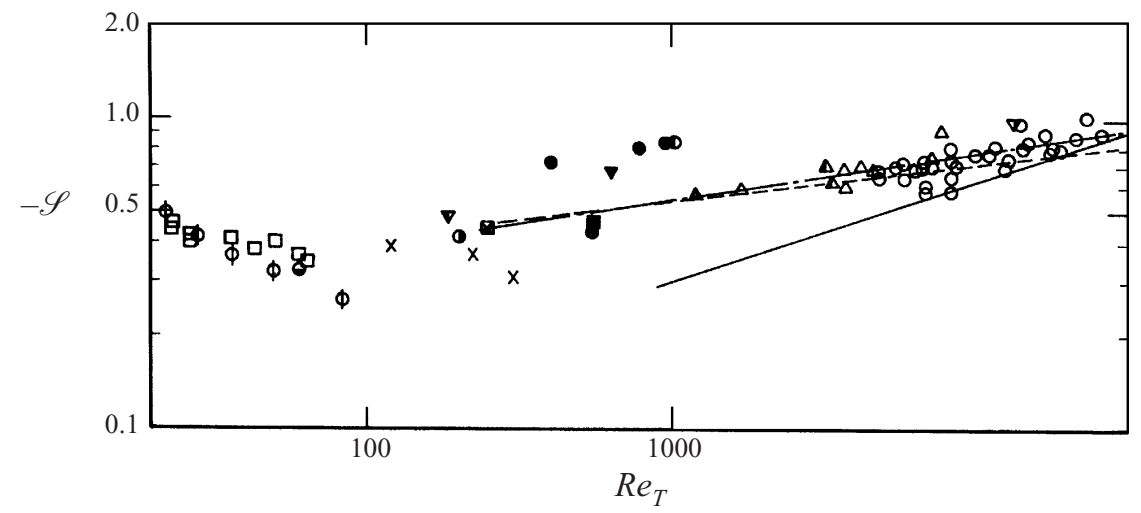

FIGURE 15. Experimental variation of skewness factor (12) with Taylor Reynolds number, in various flows (Van Atta \& Antonia 1980, figure 1; axes labels modified to conform to present nomenclature).

where $\lambda_{T}$ is the Taylor microscale, until a value of $R e_{T} \approx 70-100$, beyond which it becomes much less sensitive to Reynolds number, attaining a value of $\alpha \approx 1$ at higher Reynolds numbers. See figure 14. The value of this constant, however, may not be universal, with measurements in the range $1<\alpha<2.7$ behind non-square grids (Sreenivasan 1984). $\dagger$

A similar conclusion regarding the Taylor Reynolds number dependence of the scaled energy dissipation was documented by Jimenez et al. (1992) based on numerical simulations of turbulence in a spatially-periodic cube, in the range $36<R e_{T} \leqslant 170$. They report a value of $\alpha \simeq 0.65$, attained for $R e_{T} \geqslant 95$. Since

$$
R e_{T} \approx R e^{1 / 2}
$$

it, again, appears that $R e>R e_{\min } \approx 10^{4}$ is a necessary condition for fully-developed turbulent flow.

J. Jimenez has proposed (private communication) an alternative explanation for the Reynolds number behaviour of $\alpha(10)$ in this flow, as well as in grid turbulence, noting the decrease in the energy-spectrum wavenumber span, and, therefore, in its integral, $u^{\prime 2}$, with decreasing Reynolds number. He suggests that scaling the dissipation rate, $\varepsilon$, with an outer flow speed, $U$, instead of the r.m.s., $u^{\prime}$, could perhaps account for the observed Reynolds number behaviour.

That this neighbourhood of Taylor Reynolds numbers is special in turbulent-flow behaviour is also evident in the compilation of data by Van Atta \& Antonia (1980, figure 1), for the skewness factor,

$$
\mathscr{S} \equiv \mathscr{M}_{3}
$$

where

$$
\mathscr{M}_{n} \equiv \frac{\left\langle(\partial u / \partial x)^{n}\right\rangle}{\left\langle(\partial u / \partial x)^{2}\right\rangle^{n / 2}}
$$

$\dagger$ We note here that the proposal that $\alpha \rightarrow$ const., i.e. that the kinetic-energy dissipation should scale as,

$$
\varepsilon=\frac{v}{2}\left\langle\left(\frac{\partial u_{i}}{\partial x_{j}}+\frac{\partial u_{j}}{\partial x_{i}}\right)^{2}\right\rangle=\alpha \frac{u^{\prime 3}}{\ell},
$$

with $\alpha$ taken as a constant independent of viscosity, as originally proposed by Taylor $(1935,1937)$, is a cornerstone of turbulence theory. Its exact validity should not, however, be accepted a priori (Saffman 1968); a weak dependence on Reynolds number is (still) not excludable by available data. 
for various flows, to assess various models, including the argument by Saffman (1968, 1970 ), that, if vorticity is log-normally distributed, then $\mathscr{S} \rightarrow$ const. As can be seen, the data indicate a weak Reynolds-number dependence, at high Reynolds numbers. The slow increase in skewness, with increasing Taylor Reynolds number, does not commence until $R e_{T} \simeq 100-140$, or so (figure 15). Beyond that, the data indicate an ever-increasing intermittency, i.e. a wider (scaled) p.d.f. of velocity-gradient values, as correctly conjectured by Landau, in response to the original Kolmogorov-Oboukhov (1941) proposals, $\uparrow$ and first reported by Batchelor \& Townsend (1949). The qualitative conclusion of ever-increasing intermittency, for $R e_{T} \gtrsim 10^{2}$, is also confirmed by the data compiled by Van Atta \& Antonia (1980, figure 2), for the next velocity-gradient moment, the kurtosis, $\mathscr{K} \equiv \mathscr{M}_{4}$ (13) for various flows.

That $R e_{T}$ must exceed 100, or so, for bona-fide turbulence to be expected, has been assumed by the fluid mechanics community for some time. As articulated by Saffman (1978):

The value of $R e_{T}$ [present notation] probably has to exceed 100 to represent fully developed turbulence in which the dissipation seems to occur on scales smaller than those containing the energy.

We will return to this point below.

Experiments that included measurements of the torque in Couette-Taylor flow, in the range of Reynolds numbers of $800<R e<1.23 \times 10^{6}$ (Lathrop, Fineberg \& Swinney $1992 a, b$ ) revealed a 'well-defined, non-hysteretic transition' in a narrow range of Reynolds numbers, $10^{4}<R e_{t r}<1.3 \times 10^{4}$. The flow was found to be qualitatively different, below and above this transition, as illustrated in their flow-visualization data reproduced in figure 16, with pre- and post-transition differences reminiscent of the corresponding changes in jets (cf. figure 5). Additional flow-visualization data are available in Lathrop et al. (1992a, figures $1 a, b$ ). Beyond this transition, the dependence of the torque on Reynolds number becomes progressively weaker. Significantly, however, the torque does not attain viscosity-independent behaviour to the highest Reynolds numbers investigated (figure 17).

In thermal convection, a transition from 'soft turbulence' to 'hard turbulence' was noted for Rayleigh numbers, $R a \approx 10^{8}$, marked by a qualitative change in the p.d.f. of the measured temperature fluctuations (Heslot, Castaign \& Libchaber 1987). Since $R e \approx R a^{1 / 2}$ for the resulting turbulence in this flow (Castaign et al. 1989), we again recover a minimum Reynolds number, as required for fully-developed turbulence, of $R e \approx 10^{4}$.

In recent experiments on incompressible Richtmyer-Meshkov-instability flow, interfaces between fluids with different densities were impulsively accelerated with relatively large initial interfacial perturbations (J. W. Jacobs \& C. E. Niederhaus, unpublished work). In the resulting flow, the impulsively seeded baroclinic vorticity produces large-scale vortical structures that become turbulent when the Reynolds number, $\operatorname{Re}_{\Gamma}=\Gamma / v$, with $\Gamma$ the estimated vortex circulation, exceeds approximately $10^{4}$ (J. Jacobs, private communication). For a circular vortex, $\Gamma=(\pi / 2) U \delta$, with $U$

$\dagger$ Kolmogorov (1962) credits Landau for noting, quite soon after the original KolmogorovOboukhov similarity hypotheses were formulated, '... that they did not take into account a circumstance which arises ... from the assumption ... of the transfer of energy from the coarser vortices to the finer: with increase of the [outer-to-inner scale] ratio $L: l$, the variation of the dissipation of energy, $\epsilon, \ldots$ should increase without limit.' A footnote in the original (Russian) Landau \& Lifshitz volume Fluid Mechanics (1959), it has found its way into the main text in subsequent editions (cf. discussion in Frisch 1995). 

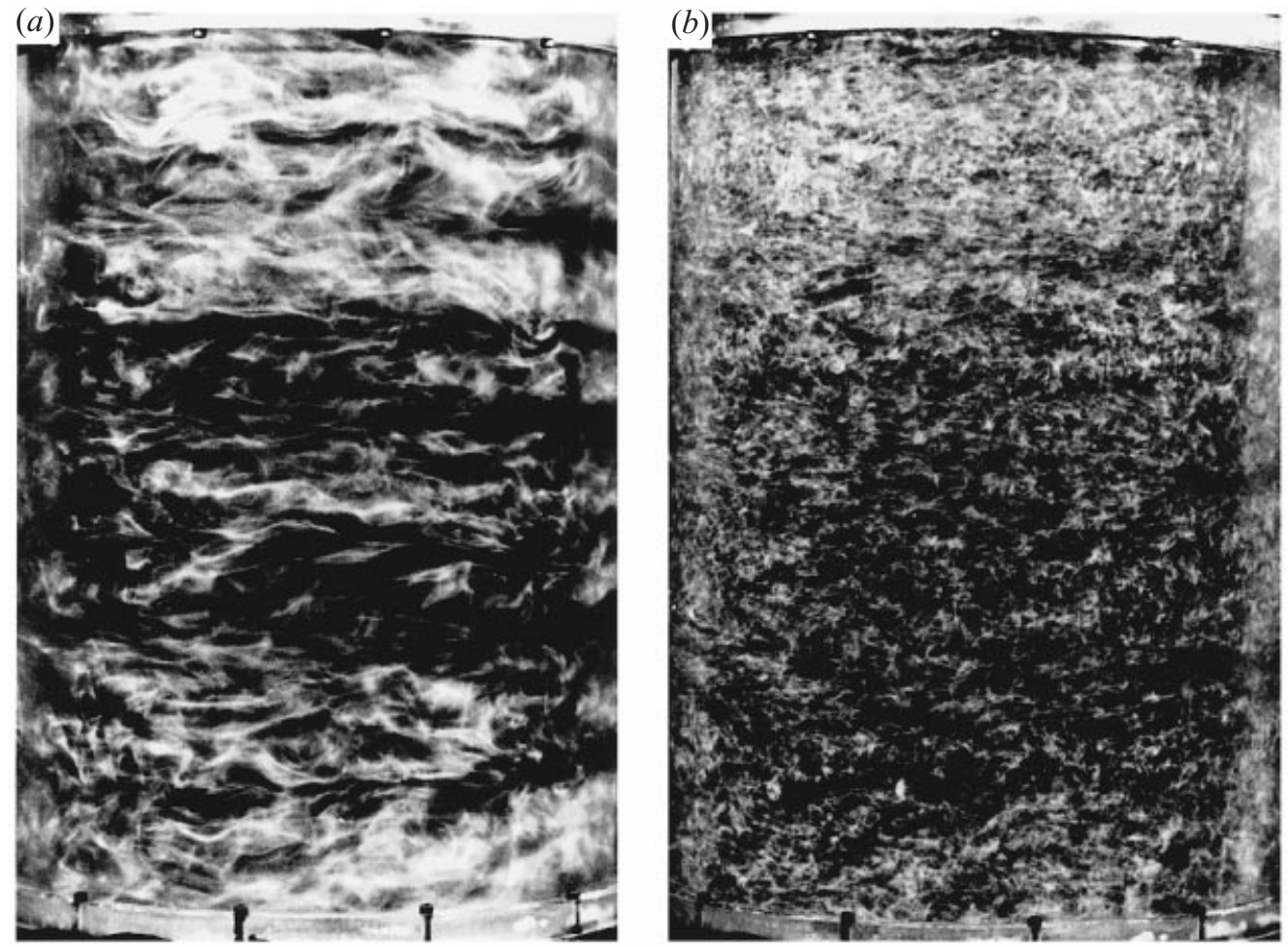

FIgURE 16. Couette-Taylor flow-visualization data at (a) $R e=0.6 \times 10^{4}$, and (b) $R e=2.4 \times 10^{4}$. From Lathrop et al. (1992b, figures $5 a, b)$. Reproduced by kind permission of Professor H. Swinney.

the velocity across the vortex core (twice the circumferential velocity at the core edge) and $\delta$ the vortex core diameter. This definition of the vortical-structure Reynolds number is close to that adopted for shear layers $(2 a)$. For shear-layer large-scale vortical structures, $\Gamma(x) \simeq \Delta U l(x)$, where $l(x) \approx 4 \delta_{\omega}(x) \simeq 2 \delta(x)$ is the large-scale-structure spacing at $x$, with $\delta_{\omega}$ the velocity-profile maximum-slope (vorticity) thickness.

\section{A criterion for fully-developed turbulence?}

As discussed in the Introduction, it has been implicitly, or explicitly, assumed for some time that the canonical attributes of turbulence are only to be expected at large Reynolds numbers. The preceding observations suggest, however, that, in addition, a rather distinct transition to a more-developed, better-mixed turbulent state occurs, within a relatively narrow range of Reynolds numbers, with a Reynolds number dependence of many flow properties that is found to be weaker beyond that.

In view of the wide variety of different flows that exhibit this behaviour, the inference can also be made that there exists a property of turbulence that induces it to transition to a well-mixed state, is associated with Reynolds numbers in excess of $R e_{\min } \approx 10^{4}$, and appears to be rather independent of the details of the flow geometry. The following is a proposal to account for this behaviour.

That this transition appears to be independent of the flow geometry suggests that the explanation may not lie with the large-scale dynamics, or the development of distinct features and organized patterns in these flows. Such attributes are, typically, 

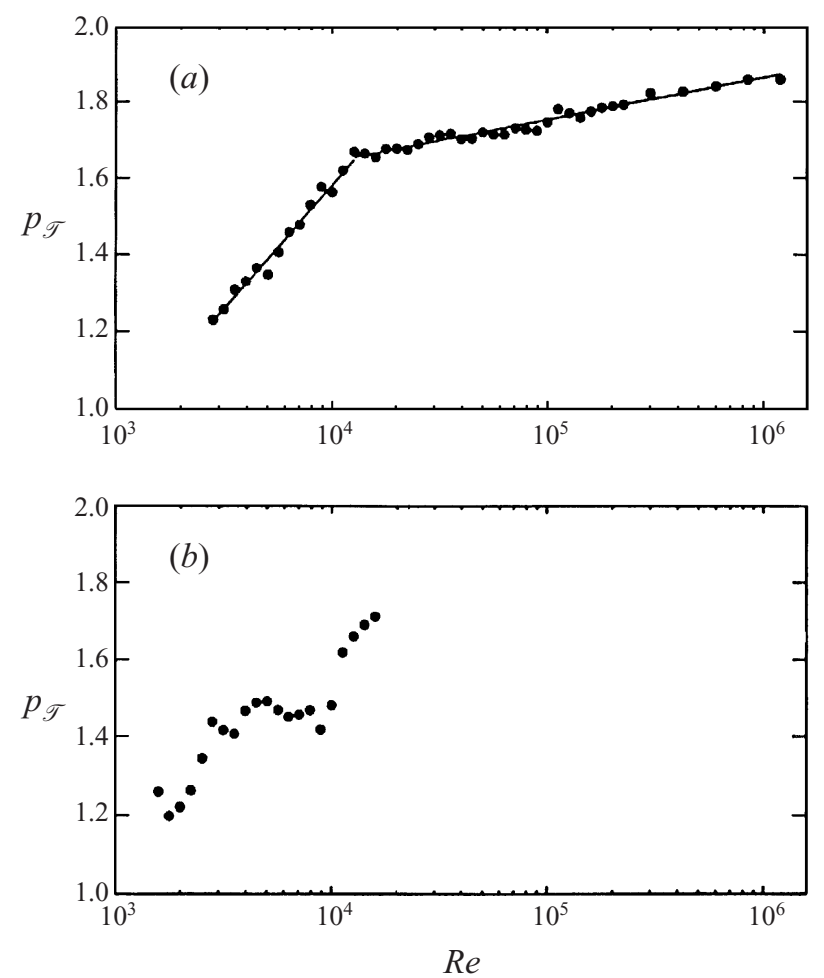

FiguRE 17. Exponent for the measured torque, $\mathscr{T}$, in Couette-Taylor flow, i.e. $p_{\mathscr{T}} \equiv$ $\partial\left(\log _{10} \mathscr{T}\right) / \partial\left(\log _{10} R e\right):(a)$ eight-vortex state, $(b)$ ten-vortex state (Lathrop et al. 1992b, figure 9; legends modified to conform to present nomenclature).

flow-geometry dependent. One is rather led to consider the significance of the various inner scales of turbulence and their Reynolds number scaling.

The 1941 Kolmogorov-Oboukhov proposals imply that the dynamics in the range of scales of size $\lambda$ that are unaffected by an outer scale, $\delta$, but are large compared to an inner, viscous-dissipation scale, which we shall take as equal to the (defined) Kolmogorov scale, i.e.

i.e. for

$$
\lambda_{K} \equiv\left(\frac{v^{3}}{\varepsilon}\right)^{1 / 4}
$$

$$
\lambda_{K} \ll \lambda \ll \delta
$$

can be treated in a universal, self-similar fashion. $\dagger$ In this range of scales, for example, the energy spectrum is predicted (and found) to exhibit a near power-law behaviour with a negative $\approx \frac{5}{3}$ exponent (e.g. Monin \& Yaglom 1975).

To refine the bounds in (15), we appreciate that independence from the dynamics of the outer scale, $\delta$, requires that an inner eddy scale, $\lambda$, should be smaller than a

$\dagger$ Kolmogorov (1962) credits Richardson with the idea on which the notion is based, namely, 'of the existence in turbulent flow of vortices on all possible scales $l<r<L\left[\lambda_{K}, \lambda\right.$, and $\delta$, respectively, in the present notation] between the 'external scale' $L$ and the 'inner scale' $l$ and of a certain uniform mechanism of energy transfer from the coarser-scaled vortices to the finer.' The attribution is, most likely, to the proposals and ideas documented in the Richardson (1922) monograph. 
scale that can be generated directly from the outer scale $\delta$. Such a scale would be estimated on the basis of an outer laminar-layer thickness, $\lambda_{L}$, that can be generated by a single $\delta$-size sweep across the whole transverse extent of the turbulent region, for example. For a laminar layer growing over a spatial extent $\delta$, we have

$$
\frac{\lambda_{L}}{\delta} \simeq c_{\mathrm{lam}} R e^{-1 / 2}
$$

The prefactor corresponding to the $99 \%$ thickness of a laminar wall (Blasius) boundary layer, for example, is given by

$$
\left(c_{\text {lam }}\right)_{\text {b.l. }} \simeq 5.0
$$

and is only a weak function of the threshold value. For the $99 \%$ thickness of a laminar shear layer (stability issues aside) with a velocity ratio, $r=U_{2} / U_{1} \simeq 0.5$ we have (e.g. Lock 1951)

$$
\left(c_{\mathrm{lam}}\right)_{r=0.5 \mathrm{~s} . \mathrm{l} .} \simeq 6.8,
$$

with values (weakly) decreasing with increasing velocity ratio, $r$. Typical internal shear layers will be characterized by lower local velocity ratios, i.e. $r \rightarrow 1$, with associated thickness prefactors that may then be assumed close to the Blasius boundary-layer value; equation $(16 b)$. Such scales are then directly connected by viscosity to the outer scale of the flow, $\delta$, in the sense that a single $\delta$-size sweep across the turbulent region will generate them through viscous action. As noted by $\mathrm{H}$. W. Liepmann in private conversation many years ago, by virtue of their dependence on Reynolds number, such scales are closely related to the Taylor microscale, $\lambda_{T}$; cf. equations $(11 a)$. For the purposes of many discussions, prefactors, such as the one in $(16 a)$, are omitted, in which case the two scales are, in fact, the same. We shall accept a generic thickness estimate for such internal laminar layers of

$$
\frac{\lambda_{L}}{\delta}=5.0 R e^{-1 / 2},
$$

dubbing the resulting thickness the Liepmann-Taylor scale.

At the other end of the spectrum, the inertial-range requirement that internal eddying motions must be inviscid dictates that their local scale $\lambda$ must be large with respect to an inner viscous scale, $\lambda_{v}$, which we may assume as proportional to the (defined) Kolmogorov dissipation scale, $\lambda_{K}(14)$. This allows the inequality that bounds the inertial range of scales (15) to be refined, i.e.

$$
\frac{\lambda_{K}}{\delta}<\frac{\lambda_{v}}{\delta}<\frac{\lambda}{\delta}<\frac{\lambda_{L}}{\delta}<1,
$$

yielding a necessary condition for fully-developed flow, also refining the ansatz articulated by Saffman (1978).

To translate this inequality to a Reynolds number requirement requires the Reynolds number dependence of the ratio of the various scales in (17) to the outer scale, $\delta$. We shall rely on $(16 d)$ for the estimate of the outer laminar-layer thickness, $\lambda_{L}$, as suggested by Liepmann. This can be compared to the Taylor scale for a turbulent jet, for example, for which direct estimates of the latter are available.

For turbulence in the far field of a jet, the Taylor scale, $\lambda_{T}$, can be estimated from the Taylor Reynolds number on the jet axis (11). This is approximately given by

$$
R e_{T} \simeq 1.4 R e^{1 / 2}
$$

(e.g. Dowling \& Dimotakis 1990; Miller \& Dimotakis 1991). Using a value of 
$u^{\prime} \simeq 0.25 u_{c l}$, on the axis of the turbulent jet, and $\delta(x) \simeq 0.4\left(x-x_{0}\right)$ for the local jet diameter, we obtain,

$$
\frac{\lambda_{T}}{\delta} \simeq 2.3 R e^{-1 / 2}
$$

which is a little smaller but close to the laminar-layer thickness, $\lambda_{L}$ (a prefactor of 2.3 for $\lambda_{T}$, vs. 5.0, for $\lambda_{L}$ ), especially considering that it is estimated from flow properties on the jet axis, where velocities are higher. A higher value would be appropriate if it were to serve as the corresponding measure throughout the jet cross section, bringing it closer to $\lambda_{L}$.

An appropriate inner viscous scale, $\lambda_{v}$, can be estimated in terms of the wavenumber $k_{v}$, where the energy spectrum deviates from the $-\frac{5}{3}$ power-law behaviour, or, $k_{v} \lambda_{K} \simeq \frac{1}{8}$ (Chapman 1979; Saddoughi 1992). This yields $\dagger$

$$
\lambda_{v} \approx \frac{2 \pi}{k_{v}} \simeq 50 \lambda_{K}
$$

accepting as an operational definition of $\lambda_{v}$ the scale where the turbulence spectrum departs from the $\approx-\frac{5}{3}$ power-law. See figure 18 .

To estimate the Reynolds number and outer-scale dependence of $\lambda_{v}$, we can use the expression from Friehe, Van Atta \& Gibson (1971), for the energy-dissipation rate on the jet axis, in the far field, i.e.

$$
\varepsilon \simeq 48 \frac{u_{j 0}^{3}}{d_{j}}\left(\frac{d_{j}}{x-x_{0}}\right)^{4}
$$

where $u_{j 0}$ is the jet-nozzle velocity, $d_{j}$ the jet-nozzle diameter, and $x_{0}$ the virtual origin of the far-field turbulent flow. Substituting in (14) we then have

$$
\frac{\lambda_{K}}{\delta} \simeq 0.95 R e^{-3 / 4}
$$

where Re here is the local-or initial, cf. $(2 b)$ - jet Reynolds number. Therefore, for a turbulent jet,

$$
\frac{\lambda_{v}}{\delta} \simeq 50 R e^{-3 / 4} .
$$

Substituting for $\lambda_{L}, \lambda_{v}$, and $\lambda_{K}$ in (17), we obtain

$$
R e^{-3 / 4}<\frac{\lambda_{v}}{\delta} \approx 50 R e^{-3 / 4}<\frac{\lambda}{\delta}<\frac{\lambda_{L}}{\delta} \approx 5.0 R e^{-1 / 2}<1 .
$$

The range of intermediate scales, i.e. scales smaller than $\lambda_{L}$ but larger than $\lambda_{v}$, can be seen to grow rather slowly with Reynolds number. Specifically, the ratio

$$
\mathscr{N}=\frac{\lambda_{L}}{\lambda_{v}}
$$

which measures the extent of the uncoupled range of spatial scales, i.e. the number of viscous scales within a Taylor scale, is given by

$$
\mathscr{N} \approx 0.1 R e^{1 / 4},
$$

$\dagger$ This calculation is discussed in Miller \& Dimotakis (1991), where, for the purpose of estimating diffusion-layer thicknesses (transition from high-to-low values of the diffusing scalar; not the full high-low-high cycle), half this estimate, i.e. $\lambda_{v} \approx \pi / k_{v} \simeq 25 \lambda_{K}$, was used. 


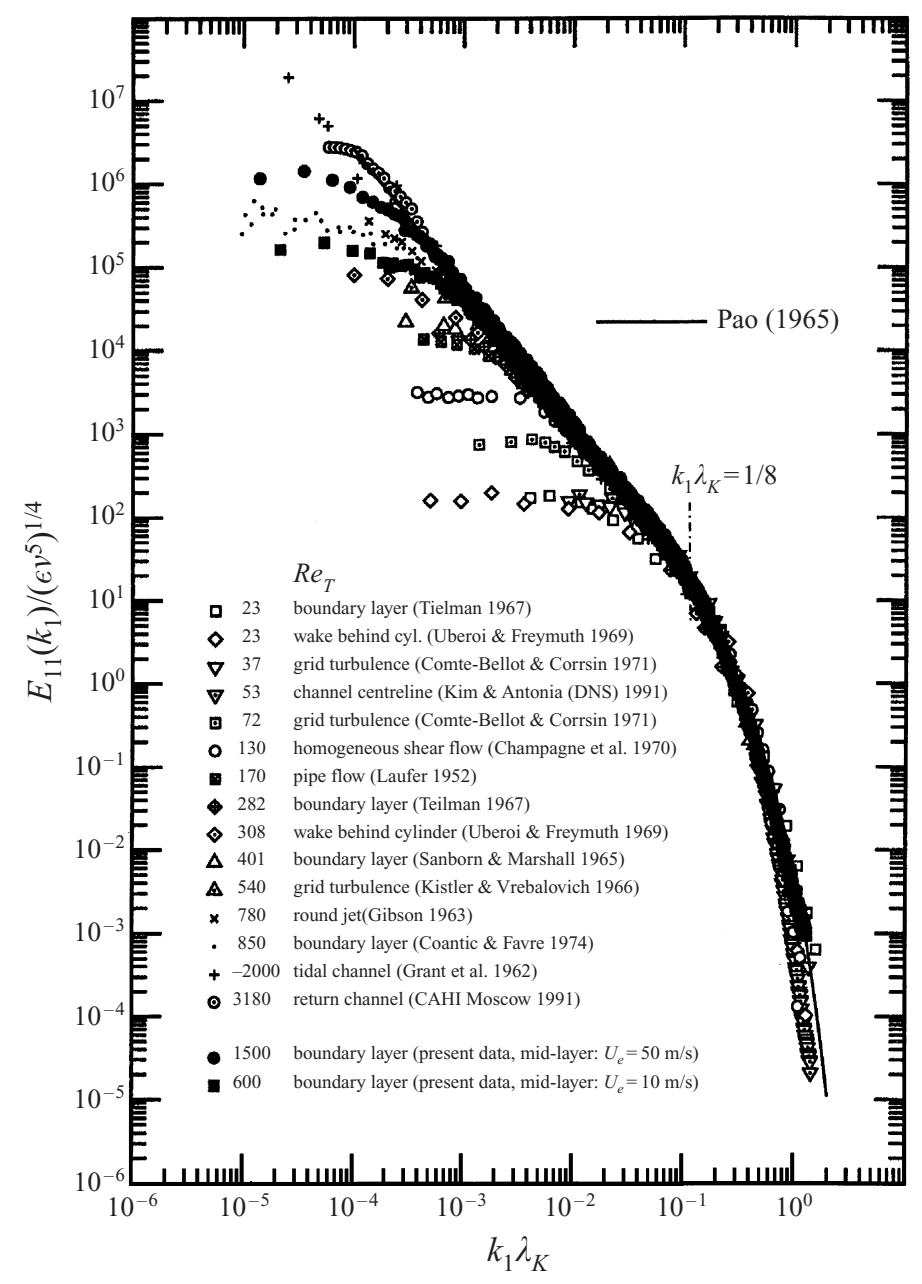

FIGURE 18. Longitudinal turbulent-velocity spectrum. Saddoughi data compilation (1992, figure 8). Legend modified to conform to present notation.

where the (approximate) prefactor of 0.1 was estimated for a turbulent jet. This is indicated schematically in figure 19. In other flows, the uncoupled range of scales can be expected to exhibit the same Reynolds number dependence, with, possibly, a different prefactor.

A similar conclusion for a minimum (Taylor) Reynolds number, stemming from a scale-separation requirement, was drawn by Pullin, Buntine \& Saffman (1994). They model turbulence as an ensemble of stretched-spiral Lundgren (1982) vortices, which are then used to calculate fine-scale turbulence quantities. Using scaling arguments, they related the size of the model vortices to the integral scale, $l$, and the Taylor scale, $\lambda_{T}$. They found that scale separation between the integral scale and the expected maximum stretched (full) length of the spiral structures requires $R e_{T} \gtrsim 10^{2}$. At lower Taylor Reynolds numbers, stretched Lundgren spirals cannot be sustained (D. Pullin, private communication).

On this basis, it can be argued that a necessary condition for fully-developed turbulence and the Kolmogorov (1941) inertial-range similarity ideas to begin to apply is the existence of a range of scales that are uncoupled from the large scales, 


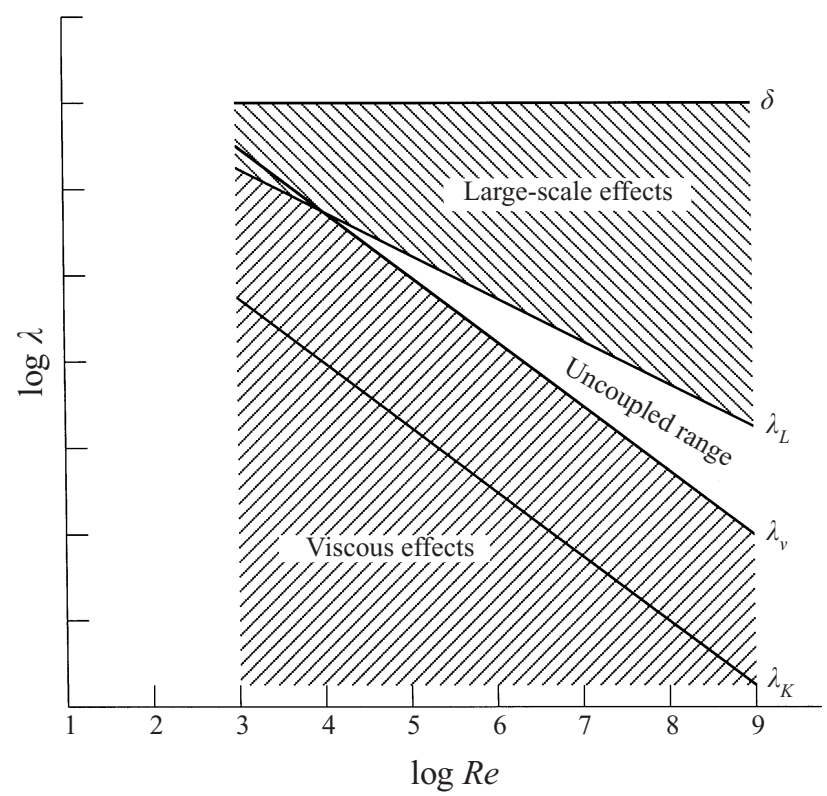

FIGURE 19. Reynolds number dependence of spatial scales for a turbulent jet.

on the one hand, and free from the effects of viscosity, on the other. Considering that we must have

$$
\frac{\lambda_{L}}{\lambda_{v}}>1,
$$

with some margin, we may infer that the existence of such a range of scales requires a minimum Reynolds number of the order of $10^{4}$, cf. $(25 b)$, or a Taylor Reynolds number of $10^{2}$, in accord with the minimum Reynolds number identified for transition to fully-developed, well-mixed turbulent flows.

\section{Discussion}

The preceding discussion supports the notion that fully-developed turbulence requires a minimum Reynolds number of order $10^{4}$, or a minimum Taylor Reynolds number of order $10^{2}$, to be sustained. These values must be viewed as a necessary, but not sufficient, condition for the flow to be in the fully developed state. The evidence suggests that both the fact that the transition occurs and the range of Reynolds number values where it occurs are universal, i.e. independent of the flow geometry. That Richtmyer-Meshkov flows as well as as accelerating, stratified shear layers (Pawlak \& Armi 1998) also exhibit this transition, at similar values of the Reynolds number, indicates that the same mechanism(s) apply, even though in the latter two cases volume sources of (baroclinic) vorticity are imporant to, or dominate, the dynamics.

This transition is different from the ones that mark transition out of a steady, laminar state, followed by a transition marked by an increase in dimensionality (two to three), then to multi-mode flow and chaotic behaviour. The criteria for those transitions can be established by stability considerations, of one kind or another, in which the stability of the flow sustained at a lower Reynolds number is altered by an increase in $R e$, i.e. 'bottom-up' transitions. Accepting the proposed mechanism of viscous decoupling of the outer and inner scales of turbulence as responsible for the 
transition criterion and the resulting minimum Reynolds number value, we see that, in contrast, the mixing transition should be viewed as a 'top-down' transition. The criteria for the latter are derived from fully developed flow, which cannot sustain that state if the outer-flow Reynolds number falls below the minimum value of $R e \simeq 1-2 \times 10^{4}$, or the Taylor Reynolds number below $R e_{T} \simeq 100-140$.

Considering the possible range of flows, the definition of the Reynolds number that should be used is an issue. The data suggest that turbulent shear flows, e.g. shear layers and jets, can be parametrized by the outer-flow Reynolds number, defined in terms of the velocity difference responsible for the shear driving the flow, e.g. $U=\Delta U=U_{1}-U_{2}$ for shear layers and the local centreline velocity, $U=u_{c l}(x)$, for jets, and the local transverse extent across which the shear is sustained, e.g. $\delta=\delta_{s l}$ for shear layers and $\delta=R_{j}(x)$, the local turbulent-region (e.g. visual) radius, for jets. If this prescription is adopted for turbulent boundary layers, the data in figure 12 suggest that if the free-stream (edge) velocity, $U=U_{e}$, and local boundary-layer $1 \%$ thickness, $\delta=\delta_{1}$ are used to define the Reynolds number, then the transition value would be somewhat higher $\left(\delta_{1}>\delta^{*}\right)$. For the Richtmyer-Meshkov flow, for which the proposed definition of Reynolds number is based on the large-scale vortical structure circulation, i.e. $R e=\Gamma / v$, the data also indicate transitional behaviour at $R e \approx 10^{4}$, with a definition that is close to the previous one, as discussed above.

There is a class of flows, however, for which an outer-scale Reynolds number, $R e$, cannot be defined. Wind-tunnel turbulence behind a grid and computed turbulence in a periodic box serve as examples. For such flows, the Taylor Reynolds number can be used, with expected transition values in the range of $R e_{T} \simeq 100-140$, based on the approximate equivalence of $R e_{T} \approx R e$ and as independently confirmed directly, as discussed above. If both outer-scale and Taylor Reynolds numbers can be defined, or are available, the two measures should yield similar results, i.e. within a factor of 2, or so.

Whereas this transition appears to be universal, how sharp this transition is, vs. Reynolds number, does appear to depend on flow details. It is remarkably sharp in the case of (Couette-Taylor) flow between concentric rotating cylinders. It is less well-defined for a shear layer and, among the flows considered, the least well-defined for turbulent jets. Perhaps an explanation for this variation lies in the definition of the Reynolds number itself (1) and the manner in which the factors that enter are imposed on each flow. For Couette-Taylor flow, both the velocity, $U=\Omega a$ and the spatial scale, $\delta=b-a$, where $\Omega$ is the differential rotation rate, with $a$ and $b$ the inner and outer cylinder radii, are well-defined by the flow boundary conditions (Lathrop et al. 1992b).

In the case of a zero-streamwise-pressure-gradient shear layer, the velocity $U=\Delta U$ is a constant and reasonably well specified by the flow boundary conditions at a particular station. The length scale $\delta=\delta(x)=\langle\delta(x, t)\rangle_{t}$, however, must be regarded as a stochastic variable in a given flow with a relatively large variance (cf. figure $1 b$ ). The Reynolds number for the shear layer is then the product of a well-defined variable and a less well-defined, stochastic variable.

In the case of a turbulent jet, both the local velocity $U=U_{j}(x)=\left\langle u_{c l}(x, t)\right\rangle_{t}$, where $u_{c l}(x, t)$ is the local jet-centreline velocity, and the length scale, $\delta=R_{j}(x)=\left\langle R_{j}(x, t)\right\rangle_{t}$, the local jet radius, must be regarded as stochastic flow variables, each with its own (large) variance. The local Reynolds number for a turbulent jet is then the product of two stochastic variables and, as a consequence, its value is the least well-defined of the three.

Viewing the Reynolds number itself as a stochastic variable, it would appear that 
the hierarchy of the sharpness of the transition to the fully-developed turbulent state may be correlated with the sharpness with which the flow and the boundary conditions allow the values of the outer-scale Reynolds number to be imposed on inner-scale dynamics.

A related issue also arises as a consequence of the definition of the local Reynolds number. As noted in the discussion of (1) and (2a), the local Reynolds number for a shear layer increases with the downstream coordinate, whereas the Reynolds number for a jet $(2 b)$ is a constant of the flow. As a consequence, a shear layer may possess regions with local Reynolds numbers below the minimum and meet the mixingtransition Reynolds number requirement within the spatial extent of a given flow, if its streamwise extent is large enough. A turbulent jet, on the other hand, will either meet the mixing-transition Reynolds number requirement over its entire downstream extent, or not. This is also relevant to the description of and dynamics in other flows.

Accepting that a near-universal Reynolds number exists for a transition into a fully developed state suggests a path for addressing at least some of the issues associated with large-eddy simulation (LES) and subgrid scale (SGS) modelling in numerical simulations of turbulent flows. To date, no clear candidate LES/SGS scheme has emerged that provides a satisfactory solution to this problem. The preceding discussion suggests that an acceptable LES/SGS model might be a direct numerical simulation (DNS) at a Reynolds number as required to exceed the mixing transition by, perhaps, not a large margin. To be sure, there is a host of Reynolds number dependent phenomena that will not be addressed by such a scheme. Nevertheless, such a simulation will represent many attributes of the turbulence sufficiently well, with no other ad hoc assumptions and models, exploiting only the documented much-weaker $R e$-dependence for $R e>1-2 \times 10^{4}$, or $R e_{T}>100-140$.

\section{Conclusions}

Data on turbulent mixing, as well as other flow phenomena, support the notion that fully-developed turbulent flow requires a minimum Reynolds number of $10^{4}$, or a Taylor Reynolds number of $R e_{T} \approx 10^{2}$, to be sustained. Conversely, turbulent flow below this Reynolds number cannot be regarded as fully developed and can be expected to be qualitatively different.

Manifestations of this transition may depend on the particular flow geometry, e.g. the appearance of streamwise vortices and three-dimensionality, in the case of shear layers. Typically, however, the transition can be identified as leading to an enhanced-mixing turbulent-flow state. That such a transition occurs, as well as the approximate Reynolds number where it is expected, appears to be a universal property of turbulence. Interestingly, whereas transition from a steady/laminar flow to unsteady/turbulent flow occurs over a relatively large range of Reynolds numbers (depending on whether viscous damping acts with the aid, or not, of proximal walls), the mixing transition to a more-developed turbulent state appears to occur within a relatively narrow range of Reynolds numbers.

These observations suggest that direct numerical simulations (DNS), performed at $R e>1-2 \times 10^{4}$, or so, may serve as good large-eddy simulation (LES) models for many turbulent flows at higher Reynolds numbers.

This paper was prepared in honour of P. G. Saffman.

I would like to acknowledge the work and discussions on this topic with P. L. Miller, and his assistance with the text, as well as the critical reading and suggestions by 
D. I. Pullin. This work was supported under AFOSR Grant Nos. F49620-94-1-0353 and F49620-98-1-0052. A first version of the data compilation and ideas presented here was documented by the author (1993) as a GALCIT Report.

\section{Note added in proof}

A. Domaradzki has brought to my attention (private communication) that the original observation by Heslot et al. (1987) that convective turbulence experiences a transition at $R a \simeq 4 \times 10^{7}$ (cf. discussion on p. 86, herein) may be attributed to a near-unity aspect ratio in those experiments. For high aspect ratios, most signs of that transition, including the change in scaling exponents, disappear, or move to much lower values of $R a$ (cf. discussion in Christie \& Domaradzki 1993, 1994).

\section{REFERENCES}

Batchelor, G. K. 1953 The Theory of Homogeneous Turbulence. Cambridge University Press.

BATCHELOR, G. K. 1959 Small-scale variation of convected quantities like temperature in turbulent fluid. Part 1. General discussion and the case of small conductivity. J. Fluid Mech. 5, 113-133.

Batchelor, G. K. \& Townsend, A. A. 1949 The nature of turbulent motion at large wave-numbers. Proc. R. Soc. Lond. A 199, 238-255.

Bernal, L. P., Breidenthal, R. E., Brown, G. L., Konrad, J. H. \& Roshko, A. 1980 On the development of three-dimensional small scales in turbulent mixing layers. Second Intl Symp. on Turbulent Shear Flows, pp. 305-313. Springer.

Bernal, L. P. \& Roshko, A. 1986 Streamwise vortex structure in plane mixing layers. J. Fluid Mech. 170, 499-525.

Bilger, R. W. 1980 Turbulent flows with nonpremixed reactants. Turbulent Reacting Flows (ed. P. A. Libby \& F. A. Williams). Topics in Applied Physics, vol. 44, pp. 65-113. Springer.

Bradshaw, P. 1966 The effect of initial conditions on the development of a free shear layer. J. Fluid Mech. 26, 225-236.

BREIDENTHAL, R. E. 1981 Structure in turbulent mixing layers and wakes using a chemical reaction. J. Fluid Mech. 109, 1-24.

BRoAdWELl, J. E. 1982 A model of turbulent diffusion flames and nitric oxide generation. Part I. TRW document 38515-6001-UT-00, EERC Final Rep. PO. 18889.

Brown, G. L. \& Rebollo, M. R. 1972 A small, fast-response probe to measure composition of a binary gas mixture. AIAA J. 10, 649-652.

Brown, G. L. \& Roshko, A. 1974 On density effects and large structure in turbulent mixing layers. J. Fluid Mech. 64, 775-816.

Castaign, B., Gunaratne, G., Heslot, F., Kadanoff, L., Libchaber, L., Thomae, S., Wu, X.-Z., ZaLeski, S. \& ZanetTI, G. 1989 Scaling of hard thermal turbulence in Rayleigh-Bénard convection. J. Fluid Mech. 204, 1-30.

Catrakis, H. J. \& Dimotakis, P. E. 1996 Mixing in turbulent jets: scalar measures and isosurface geometry. J. Fluid Mech. 317, 369-406 (referred to herein as CD96).

Chapman, D. R. 1979 Computational aerodynamics development and outlook. AIAA J. 17, 12931313.

Christie, S. L. \& Domaradzki, J. A. 1993 Numerical evidence for nonuniversality of the soft/hard turbulence classification for thermal convection. Phys. Fluids A 5, 412-421.

Christie, S. L. \& DomaradzKi, J. A. 1994 Scale dependence of the statistical character of turbulent fluctuations in thermal convection. Phys. Fluids 6, 1848-1855.

Coles, D. 1956 The law of the wake in the turbulent boundary layer. J. Fluid Mech. 1, 191-226.

Coles, D. 1968 The young person's guide to the data Proc. AFOSR-IFP-Stanford Conference Computation of Turbulent Boundary Layers III, (ed. D. Coles \& Hirst), pp. 1-45.

Collins, D. J., Coles, D. E. \& Hicks, J. W. 1978 Measurements in the turbulent boundary layer at constant pressure in subsonic and supersonic flow. Part I. Mean flow. AEDC-TR-78-21.

Corrsin, S. 1951 On the spectrum of isotropic temperature fluctuations in isotropic turbulence. $J$. Appl. Phys. 22, 469-473. 
Dahm, W. J. A. \& Dimotakis, P. E. 1987 Measurements of entrainment and mixing in turbulent jets. AIAA J. 25, 1216-1223.

Dahm, W. J. A., Dimotakis, P. E. \& Broadwell, J. E. 1984 Non-premixed turbulent jet flames. AIAA Paper 84-0369.

Dimotakis, P. E. 1984 SF $_{6} \rightarrow$ Li combustor. Garrett Interim Report (20 January 1984), PO. 0044-323.

Dimotakis, P. E. 1986 Two-dimensional shear-layer entrainment. AIAA J. 24, 1791-1796.

Dimotakis, P. E. 1991 Turbulent free shear layer mixing and combustion. High Speed Flight Propulsion Systems. Progress in Astronautics and Aeronautics, vol. 137, ch. 5, 265-340.

Dimotakis, P. E. 1993 Some issues on turbulent mixing and turbulence. GALCIT Rep. FM93-1a.

Dimotakis, P. E. \& Brown, G. L. 1976 The mixing layer at high Reynolds number: Large-structure dynamics and entrainment. J. Fluid Mech. 78, 535-560 + 2 plates.

Dimotakis, P. E., Miake-Lye, R. C. \& Papantoniou, D. A. 1983 Structure and dynamics of round turbulent jets. Phys. Fluids 26, 3185-3192.

Dowling, D. R. 1988 Mixing in gas phase turbulent jets. PhD thesis, California Institute of Technology.

Dowling, D. R. \& Dimotakis, P. E. 1990 Similarity of the concentration field of gas-phase turbulent jets. J. Fluid Mech. 218, 109-141.

ECKART, C. 1948 An analysis of the stirring and mixing processes in incompressible fluids. J. Mar. Res. VII, 265-275.

Friehe, C. A., Van Atta, C. W. \& Gibson, C. H. 1971 Jet turbulence: Dissipation rate measurements and correlations. AGARD Turbulent Shear Flows CP-93, 18.1-7.

FrISCH, U. 1995 Turbulence. The Legacy of Kolmogorov. Cambridge University Press.

GiLbReCH, R. J. 1991 An experimental investigation of chemically-reacting, gas-phase turbulent jets. $\mathrm{PhD}$ thesis, California Institute of Technology.

Gilbrech, R. J. \& Dimotakis, P. E. 1992 Product formation in chemically-reacting turbulent jets. AIAA Paper 92-0581.

Goldstein, S. 1938 Modern Developments in Fluid Dynamics. Clarendon. (Reprinted 1965, Dover publications.)

Hammer, J. A. 1993 Lifted turbulent jet flames. PhD thesis, California Institute of Technology.

Heslot, F., Castaign, B. \& Libchaber, A. 1987 Transitions to turbulence in helium gas. Phys. Rev. A 36, 5870-5873.

HinZE, J. O. 1975 Turbulence, 2nd Edn. McGraw-Hill.

HotтeL, H. C. 1953 Burning in laminar and turbulent fuel jets. Fourth Symp. (Intl) on Combustion, pp. 97-113. Williams and Wilkins, Baltimore.

HuANG, L.-S. \& Ho, C.-M. 1990 Small-scale transition in a plane mixing layer. J. Fluid Mech. 210, $475-500$.

Jimenez, J., Martinez-Val, R. \& Rebollo, M. 1979 On the origin and revolution of threedimensional effects in the mixing layer. U. Politec. Madrid Internal Rep. DA-ERO-70-G-079.

Jimenez, J., Wray, A. A., Saffman, P. G. \& Rogallo, R. S. 1992 The structure of intense vorticity inhomogeneous isotropic turbulence. Studying Turbulence Using Numerical Simulation Database-IV, Proc. 1992 Summer Program, pp. 21-45. (Center for Turbulence Research, NASA Ames \& Stanford U.)

Kolmogorov, A. N. 1941a Local structure of turbulence in an incompressible viscous fluid at very high Reynolds numbers. Dokl. Akad. Nauk SSSR 30, 299.

Kolmogorov, A. N. 1941 Dissipation of energy in locally isotropic turbulence. Dokl. Akad. Nauk. SSSR. 66, 825.

Kolmogorov, A. N. 1962 A refinement of previous hypotheses concerning the local structure of turbulence in a viscous incompressible fluid at high Reynolds number. J. Fluid Mech. 13, $82-85$.

KonRaD, J. H. 1976 An experimental investigation of mixing in two-dimensional turbulent shear flows with applications to diffusion-limited chemical reactions. $\mathrm{PhD}$ thesis, California Institute of Technology.

Koochesfahani, M. M. \& Dimotakis, P. E. 1986 Mixing and chemical reactions in a turbulent liquid mixing layer. J. Fluid Mech. 170, 83-112 (referred to herein as KD86).

Kuo, K. K. 1986 Principles of Combustion. John Wiley. 
Landau, L. D. \& Lifshitz, E. M. 1959 Course of Theoretical Physics, Vol. 6. Fluid Mechanics. Pergamon.

Lathrop, D. P., Fineberg, J. \& Swinney, H. L. $1992 a$ Turbulent flow between Concentric Rotating Cylinders at Large Reynolds Number. Phys. Rev. Lett. 68, 1515-1518.

Lathrop, D. P., Fineberg, J. \& Swinney, H. L. $1992 b$ Transition to shear-driven turbulence in Couette-Taylor flow. Phys. Rev. A 46, 6390-6405.

LiEPMANn, D. \& GHARIB, M. 1992 The role of streamwise vorticity in the near-field entrainment of round jets. J. Fluid Mech. 245, 643-668.

Lock, R. C. 1951 The velocity distribution in the laminar boundary layer between parallel streams. Q. J. Mech. Appl. Math. IV, 42-63.

LundGRen, T. S. 1982 Strained spiral vortex model for turbulent fine structure. Phys. Fluids 25, 2193-2203.

Masutani, S. M. \& Bowman, C. T. 1986 The structure of a chemically reacting plane mixing layer. J. Fluid Mech. 172, 93-126.

Miller, P. L. 1991 Mixing in high Schmidt number turbulent jets. PhD thesis, California Institute of Technology.

Miller, P. L. \& Dimotakis, P. E. 1991 Reynolds number dependence of scalar fluctuations in a high Schmidt number turbulent jet. Phys. Fluids A 3, 1156-1163.

Monin, A. S. \& Yaglom, A. M. 1975 Statistical Fluid Mechanics: Mechanics of Turbulence II (ed. J. Lumley). MIT Press.

Moser, R. D. \& Rogers, M. M. 1991 Mixing transition and the cascade to small scales in a plane mixing layer. Phys. Fluids A 3, 1128-1134.

Mungal, M. G. \& Dimotakis, P. E. 1984 Mixing and combustion with low heat release in a turbulent mixing layer. J. Fluid Mech. 148, 349-382.

Namazian, M. \& Kelly, J. T. 1988 Near-field instantaneous flame and fuel concentration structures. Twenty-Second Symp. (Intl) on Combustion, pp. 627-634. The Combustion Institute.

Norberg, C. \& Sundén, B. 1987 Turbulence and Reynolds number effects on the flow and fluid forces on a single cylinder in cross flow. Trans. AMSE: J. Fluid Engng 1, 337-357.

Oboukhov, A. M. 1941 On the distribution of energy in the spectrum of turbulent flow. C. R. Acad. Sci. URSS 32, 19, and Izv. Akad. Nauk. SSSR, Geogr. i. Goefiz. 5, 453.

Oвоuкhov, A. M. 1949 Structure of the temperature field in turbulent flows. Izv. Ak. Nauk SSR, Geogr. Geophys. 13, 58-69.

PaWlak, G. \& Armi, L. 1998 Vortex dynamics in a spatially accelerating shear layer. J. Fluid Mech. 376, $1-35$.

Pullin, D. I., Buntine, J. D. \& Saffman, P.G. 1994 On the spectrum of a stretched spiral vortex. Phys. Fluids 6, 3010-3027.

Richardson, L. F. 1922 Weather Prediction by Numerical Process. Cambridge University Press.

Roshko, A. 1990 The mixing transition in free shear flows. The Global Geometry of Turbulence (Impact of nonlinear dynamics), NATO Advance Research Workshop, 8-14 July 1990, Rota (Cádiz), Spain.

Roshko, A. 1993 Perspectives on bluff body aerodynamics. J. Wind Engng Indust. Aerodyn. 49, $79-100$.

Saddoughi, S. G. 1993 Local isotropy in high Reynolds number turbulent shear flows. Annual Research Briefs - 1992, Center for Turbulence Research, NASA Ames \& Stanford University, pp. 237-262.

Saffman, P. G. 1968 Lectures on Homogeneous Turbulence. Topics in Non-Linear Physics (ed. N. Zabusky), pp. 485-614. Springer.

SAFFman, P. G. 1970 Dependence on Reynolds number of high-order moments of velocity derivatives in isotropic turbulence. Phys. Fluids 13, 2192-2193.

SaffMAN, P. G. 1978 Problems and progress in the theory of turbulence. Structure and Mechanisms of Turbulence II. Lecture Notes in Physics, vol. 76 (ed., H. Fiedler), pp. 273-306. Springer.

Seitzman, J., Üngüt, A., Paul, P. \& Hanson, R. 1990 Imaging and characterization of $\mathrm{OH}$ structures in a turbulent nonpremixed flame. Twenty-third Symp. (Intl) on Combustion, pp. 637-644. The Combustion Institute, Pittsburgh.

Slessor, M. D., Bond, C. L. \& Dimotakis, P. E. 1998 Turbulent shear-layer mixing at high Reynolds numbers: effects of inflow conditions. J. Fluid Mech. 376, 115-138. 
Sreenivasan, K. R. 1984 On the scaling of the turbulent energy dissipation rate. Phys. Fluids 27, $1048-1050$.

TaYlor, G. I. 1935 Statistical theory of turbulence. Part I. Proc. R. Soc. Lond. A 151, 421-444.

TAYLOR, G. I. 1937 Statistical theory of isotropic turbulence. J. Aeronaut. Sci. IV, 311-319.

THORPE, S. 1971 Experiments on instability and turbulence of stratified shear flows: miscible fluids. J. Fluid Mech. 46, 299-319.

THORPE, S. 1973 Experiments on instability and turbulence in a stratified shear flow. J. Fluid Mech 61, 731-751.

Van Atta, C. W. \& Antonia, R. A. 1980 Reynolds number dependence of skewness and flatness factors of velocity derivatives. Phys. Fluids 23, 252-257.

WedDELl, D. 1941 Turbulent mixing in gas flames. PhD thesis, Massachusetts Institute of Technology.

Wygnanski, I. \& Champagne, F. H. 1973 On transition in a pipe. Part I. The origin of puffs and slugs and the flow in a turbulent slug. J. Fluid Mech. 59, 281-335. 